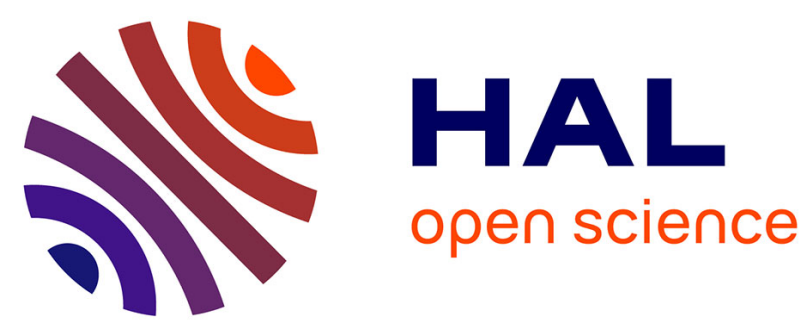

\title{
Temperature and chemical composition of droplets by optical measurement techniques: a state-of-the-art review
}

Fabrice Lemoine, Guillaume Castanet

\section{To cite this version:}

Fabrice Lemoine, Guillaume Castanet. Temperature and chemical composition of droplets by optical measurement techniques: a state-of-the-art review. Experiments in Fluids, 2013, 54 (7), pp.1572. 10.1007/s00348-013-1572-9 . hal-01431048

\section{HAL Id: hal-01431048 \\ https://hal.univ-lorraine.fr/hal-01431048}

Submitted on 3 Apr 2017

HAL is a multi-disciplinary open access archive for the deposit and dissemination of scientific research documents, whether they are published or not. The documents may come from teaching and research institutions in France or abroad, or from public or private research centers.
L'archive ouverte pluridisciplinaire HAL, est destinée au dépôt et à la diffusion de documents scientifiques de niveau recherche, publiés ou non, émanant des établissements d'enseignement et de recherche français ou étrangers, des laboratoires publics ou privés.

\section{(ㅇ)(1) $\$$}

Distributed under a Creative Commons Attribution - NonCommercial - NoDerivatives 44.0 


\title{
Temperature and chemical composition of droplets by optical measurement techniques: a state-of-the-art review
}

\author{
Fabrice Lemoine - Guillaume Castanet
}

\begin{abstract}
The measurement of the sizes and the velocities of droplets relies upon widespread and well-established techniques, but characterizing their temperature and their composition remains challenging. The lack of standard methods is particularly detrimental, given the importance of these parameters for validating models and numerical simulations of many spray processes. Heat and mass transfers are dominant aspects in applications such as spray combustion in IC engines, spray cooling, spray drying, wet scrubbers in which liquid sprays capture gas pollutants and also the preparation of nanoparticles via spray route. This paper provides a review of the main techniques available to optically measure the temperature and chemical compositions of single droplets and sprays. Most of these techniques are based on phenomena related to light interaction with matter. Photoluminescence processes like fluorescence and phosphorescence have temperature and composition dependences which can be exploited, while other methods rely on light scattering by the droplets. In particular, the angular position of the rainbow is very sensitive to the refractive index and then to both the temperature and composition. Less widely used diagnostic methods include Raman scattering, thermochromic liquid crystals, thermographic phosphors, infrared thermography, morphology-dependent resonances and their subsequent effects on the stimulated emission of dye molecules. In this review, the emphasis
\end{abstract}

F. Lemoine - G. Castanet

Université de Lorraine, LEMTA, UMR 7563,

Vandoeuvre-lès-Nancy, France

F. Lemoine $(\square) \cdot$ G. Castanet

CNRS, LEMTA, UMR 7563, Vandoeuvre-lès-Nancy, France

e-mail: fabrice.lemoine@ensem.inpl-nancy.fr is mainly placed on two groups of techniques-methods based on laser-induced fluorescence and those based on light scattering-but details about alternative methods will be also provided. The potential of combining fluorescence-based techniques or rainbow refractometry with a droplet sizing measurement technique to derive temperature and composition per size class will be also discussed.

\section{Introduction}

The measurement of the sizes and the velocities of droplets relies upon widespread and well-established techniques, but characterizing their temperature and their composition remains challenging. The lack of standard methods is particularly detrimental, given the importance of these parameters for validating models and numerical simulations of many processes involving sprays.

Measuring the droplet temperature and composition is required to investigate heat and mass transfers at different scales. For the purposes of many fundamental studies, it is of interest to characterize phenomena which occur at the droplet scale. Some of these studies deal with motionless droplets which can be suspended on a wire (Saharin et al. 2012), levitated by means of optical tweezers (Ashkin 1970), ultrasonic acoustic fields (Tuckermann et al. 2002) or electrodynamic quadrupoles (Heinisch et al. 2009). Single moving droplets and streams of monodisperse droplets formed through a continuous jet breakup are also frequently used for studying the elementary properties of droplets (König et al. 1986). Large scale measurements in sprays have been reported in a wide range of studies some of which covered areas close to industrial applications. Spray measurements generally require calculation of the 
average spatial and/or temporal properties of the droplets because many droplets will cross the probe volume.

Heat and mass transfers play a dominant role in many industrial applications of sprays. With internal combustion engines, spray studies are of interest for research into potential emissions reductions and the overall optimization of combustion. Modeling droplet heating and evaporation as well as fuel-air mixing (Abramzon and Sirignano 1989; Sazhin et al. 2006) requires advanced experimentally obtained knowledge of droplet behavior, and temperature and composition measurements are particularly relevant parameters which need to be characterized. One issue of particular interest is the change in composition of the droplets due to the preferential evaporation of the more volatile chemical species contained in the fuel which are typically made up of hundreds of chemical species. This differential evaporation both affects the liquid phase (Maqua et al. 2007; Sazhin et al. 2010; Tong and Sirignano 1986) and has a direct influence on vapor composition and thus on the kinetics and extents to which chemical reactions occur in the gas phase.

Measuring droplet temperature is also of great interest in research studies related to spray cooling for applications in the steel processing industry (Castanet et al. 2009; Panao and Moreira 2009) or in the heat management of increasingly high-speed electronic components (Kim 2007). For these spray applications to succeed, the capacity to remove high heat fluxes from the surface of the hot wall is required. In Dunand et al. (2013), temperature measurements of droplets impinging a hot wall provided insight into the contribution of liquid heating to the cooling rate of the wall.

For certain applications, droplets can be considered almost perfect chemical micro-reactors. As the only contact surface is the liquid/gas interface, they offer the advantage of avoiding solid walls around the sample which avoids any chemical and thermal contamination by a container (Omrane et al. 2004b). The flow circulation inside the droplet and the small volume of the droplet promote a rapid mixing of the reagents (Teh et al. 2008). The large area/volume ratio of the droplets enhances surface phenomena effects, particularly evaporation and heating which are of importance in some conversion processes like spray drying. In spray drying, temperature and concentration changes at the droplet surface trigger chemical reactions and phase changes like crystallization inside the droplets (Saha et al. 2010a) and modifications of the droplet shape and structure (for example by agglomeration of the solid beads in the slurry). Spray drying enables the production of a dry powder from a liquid or slurry containing macroscopic aggregates and nanoparticles (Wu et al. 1987). For many years, this has been an intensive area of research because it is one of the preferred methods for drying many thermally sensitive materials such as foods, pharmaceuticals, cosmetics. Information about the composition of both liquid and solid phases is essential when investigating spray drying processes (Seydel et al. 2006; Yarin et al. 2002). Liquid sprays are also used in wet scrubbers to capture gaseous and particulate pollutants. When fine droplets are atomized in spray towers, this provides a large liquid-gas interface for absorbing gas and which maximizes the number of droplet impacts with the pollutant particles. Controlling the droplet composition non-intrusively appears a major concern in all these applications which involve droplets undergoing a chemical modification. The temperature of the droplets is also of significant interest because since chemical kinetics and equilibrium, absorption and diffusion coefficients are all thermally dependent.

This paper presents a review of the main optical techniques devised to measure the temperature and the chemical compositions of droplets non-intrusively. Most of them are based on phenomena related to light interaction with matter which are influenced to a certain extent by the temperature or the chemical composition of the investigated medium. These techniques share a common feature in that they all provide indirect measurements.

A first group of measurement techniques relies on photoluminescence processes like fluorescence or phosphorescence which are the consequence of the spontaneous relaxation of molecular species after these have been excited by a type of energy. If a laser is used to populate the excited state, the method is called laser-induced fluorescence or phosphorescence. The emitted radiation has a temperature and possibly a composition dependence that can be exploited, when it is known from a model or an empirical calibration.

In fluorescence-based diagnostics, an organic dye chosen for the high sensitivity of its emission to the temperature is generally added to the liquid when the probed parameter is the droplet temperature. Alternatively, thermographic phosphors in the form of fine powders can be added to the liquid and efficiently emit a light that also changes with temperature. When the probed parameter is the composition, the fluorescent molecules need to be a chemical species which is naturally present in the multicomponent droplet.

Single-color intensity-based methods can be used in principle, but their practical implementation is not straightforward in the case of droplets. The main problem with these methods is that any modification of the signal intensity which does not exclusively originate from temperature or composition variations must be accounted for separately. As ratiometric approaches do not present these drawbacks, they are generally preferred for measuring droplet temperature. However, intensity ratios have been 
found to cancel out the influence of the concentration on the fluorescence signal almost totally. One possibility is to use a single dye with two spectral bands of detection. Laser-induced exciplex fluorescence has been also reported as a means to probe droplet temperature based on the ratio of excimer/monomer fluorescence intensities.

Measuring the lifetime of fluorescence and phosphorescence can be an alternative for researches aiming to characterize the temperature and composition of droplets because such measurements provides information about the physical and chemical environment of the emitting molecules. As it is an intrinsic parameter, lifetime measurements inherently do not require the same care as intensitybased methods. The decay time can be determined using both time and frequency-domain methods. Time-resolved measurements enable more of the signature of a compound to be elucidated in the case of a mixture of several fluorescent or phosphorescent molecules. However, timeresolved measurements are instrumentally sophisticated as they require fast detection systems and light pulses whose durations are short in comparison with the excited state lifetime. These technical considerations may partially explain the limited number of time-resolved fluorescence lifetime measurements reported so far in the field of droplets given that fluorescence is very short-lived (on the nanosecond timescale). In comparison, phosphorescence is slow (often milliseconds or longer) and therefore timeresolved measurements can be easily employed, particularly gating approaches. However, quenching by impurities especially oxygen usually makes phosphorescence difficult to observe. Phosphors are noticeable in that their quenching is not affected by the molecules present in the medium in which they are dispersed.

Other measurement techniques rely on elastic or inelastic light scattering by the droplets or by the molecules of certain chemical species present in the droplet. In elastic light scattering, the wavelength is not changed by scattering and the scattered light intensity is only a function of the scattering angle. This phenomenon is the most rigorously described by the Mie theory in the size range of the droplets in most applications. Characteristic features of the light scattered by the droplets, in particular the angular position of the rainbow, are very sensitive to the refractive index and thus the temperature and composition of the droplet. While standard rainbow refractometry is dedicated to the characterization of single droplets and requires perfectly spherical droplets, global rainbow refractometry applies to an ensemble of droplets and can be used if some of them are non-spherical as long as they are randomly oriented. A particular issue for the implementation of this technique is the robustness of the signal inversion algorithm as this requires knowing the droplet size distribution. Measurement reliability is also affected by refractive index gradients inside the droplet, and these are unavoidable in the case of evaporating single or multicomponent droplets. Finally, the fact that it is impossible to separate the effect of temperature or composition variations in the refractive index measurements is another difficulty involved with using this technique.

Raman scattering is a form of inelastic scattering of molecules which can be exploited to characterize the chemical composition of a medium and in some cases its temperature by using both the surface area and the position of the peaks in the Raman spectrum. Spontaneous Raman scattering has been utilized in single-phase liquid flows and in droplet flows. The main drawback of this method is the weakness of the signal which would in practice require high power lasers being used. However, coupling Raman emission and morphology-dependent resonances in droplets lead to Raman stimulated emission which greatly improves the method's capabilities - the Raman signal is significantly amplified, and the sensitivity in the composition variation becomes exponential.

Finally, alternative diagnostics including thermochromic liquid crystals, infrared thermography, morphology-dependent resonances will be also presented.

\section{Measurement techniques based on light scattering}

\subsection{Rainbow refractometry}

Rainbow refractometry is a non-intrusive technique that simultaneously provides information about the refractive index and droplets size. The droplet temperature and composition can be inferred from the refractive index provided that the dependence of the refractive index on both temperature and composition is known. Standard rainbow refractometry (SRR) applies to single droplets and global rainbow refractometry (GRR) to an ensemble of droplets contained in an extended probe volume.

\subsubsection{Standard rainbow refractometry (SRR)}

A first approximation of the path of light through a droplet can be obtained by applying the laws of geometrical optics. A sketch of the different modes of rays resulting from the interaction of light with a transparent spherical particle is given in Fig. 1. Each time light strikes the surface of the particle, part of the light is reflected and part is refracted. Rays reflected directly from the surface are labeled as order 0 , while those transmitted directly through the droplet are designated order 1 . The rays of order 2 emerge after one internal reflection and it is these rays which give rise to the primary rainbow. The superimposition of all the order 2 rays shows that these rays emerge from the particle with 
the greatest concentration in the vicinity of a specific scattering angle called the rainbow angle. The enhancement in the intensity of the scattered light in this angular region corresponds to the primary rainbow. Higher-order rainbows (i.e., made up of rays of higher orders) can be also observed. According to van de Hulst (1981), following the assumptions of the geometrical optics, the angle $\theta_{\mathrm{rg}}$ of the primary rainbow can be expressed as:

$\theta_{r g}=4 \arcsin \left(\frac{1}{n} \sqrt{\frac{4-n^{2}}{3}}\right)-2 \arcsin \left(\sqrt{\frac{n^{2}-1}{3}}\right)$,

where $n$ is the refractive index of the liquid of interest. Basically, SRR consists of detecting the light scattered by a single transparent particle to determine the angular position of the rainbow and thus the refractive index from Eq. (1). However, geometrical optics do not account for diffraction and interference of light. Airy's theory of rainbow addresses this issue by considering the wave nature of light (Airy 1838) thus showing that the rainbow angle $\theta_{r a}$ or more precisely the angular position of the first maximum

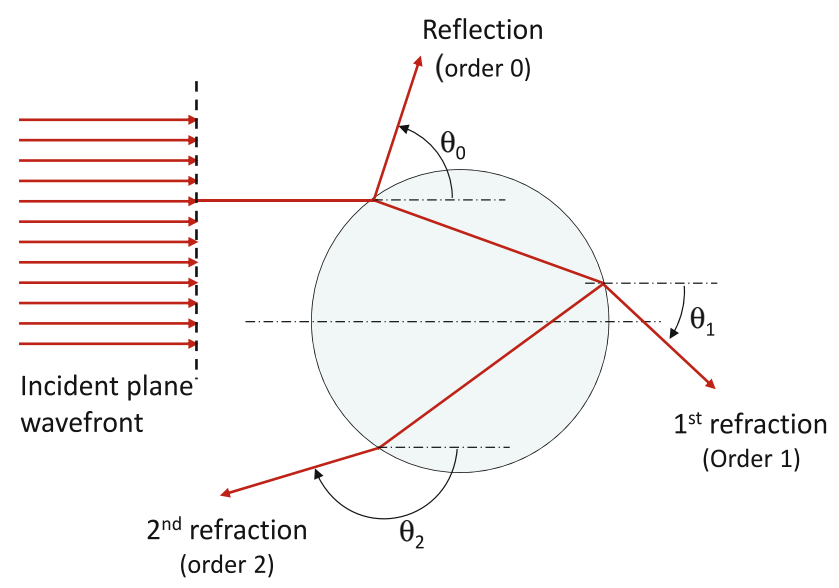

Fig. 1 Interaction of light with a spherical droplet according to geometrical optics of the primary rainbow pattern is a decreasing function of the droplet size:

$\theta_{r a}=\theta_{r g}+\frac{1.0873}{\sqrt{\left(n^{2}-1\right) / 3}}\left(\lambda^{2} \frac{\sqrt{\left(4-n^{2}\right) / 3}}{16 D^{2}}\right)$,

where $\lambda$ is the light wavelength and $D$ is the droplet diameter. Airy's theory relies on the assumption that the intensity of light in a rainbow could be modeled using a plane wave-front. This approximation means that it may suffer from noticeable discrepancies especially at small droplet sizes (Lee 1998; van de Hulst 1981). The LorenzMie theory (LMT) provides a solution to Maxwell's equation for the scattering of electromagnetic radiation by a sphere and therefore applies to particles of small size parameter.

The first published results concerning SRR were reported by Massoli et al. (1993), Roth et al. (1988, 1991), Sankar et al. (1993) and Van Beeck and Riethmuller (1994, 1995). In a SRR setup, a CW laser illuminates a single droplet or an ensemble of droplets to produce rainbows which are monochromatic (Fig. 2). Spatial filtering may be required to simultaneously select single droplets when the method is applied to a spray. The $\mathrm{CW}$ laser may be replaced advantageously by a pulsed laser which allows the rainbow pattern to be frozen to eliminate perturbations from transient events such as oscillations of the droplet shape (van Beeck and Riethmuller 1998). A CCD camera imaging the focal plane of a lens can be used to observe the angular distribution of the scattered light in the vicinity of the rainbow angle.

A typical image of the rainbow pattern is presented in Fig. 3. The rainbow is composed of a low-frequency structure, the Airy fringes which are perfectly visible in Fig. 3 and in the intensity profile displayed Fig. 4. Airy fringes originate from interference between rays of order 2 which have experienced one internal reflection. These rays emerge from the droplet in the same angular direction but

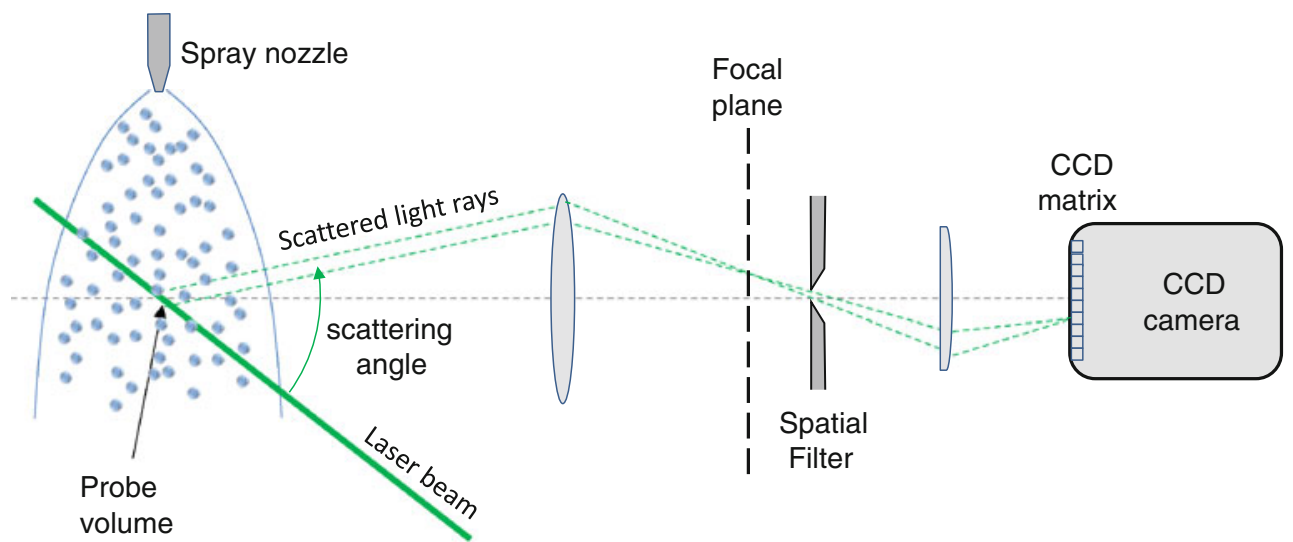

Fig. 2 Typical standard rainbow refractometry setup 


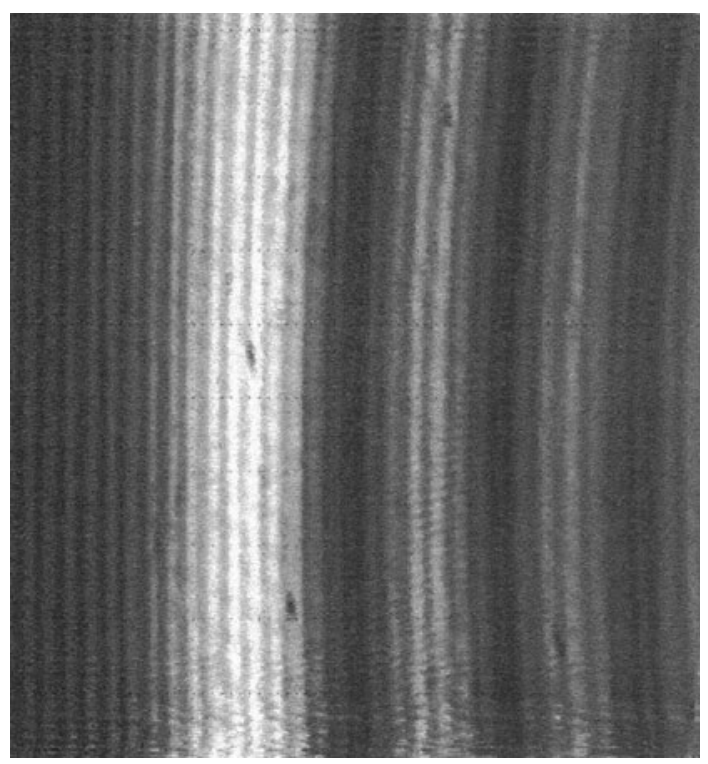

Fig. 3 Standard rainbow pattern obtained from a single droplet in a water spray. Reprinted from van Beeck et al. (2003)

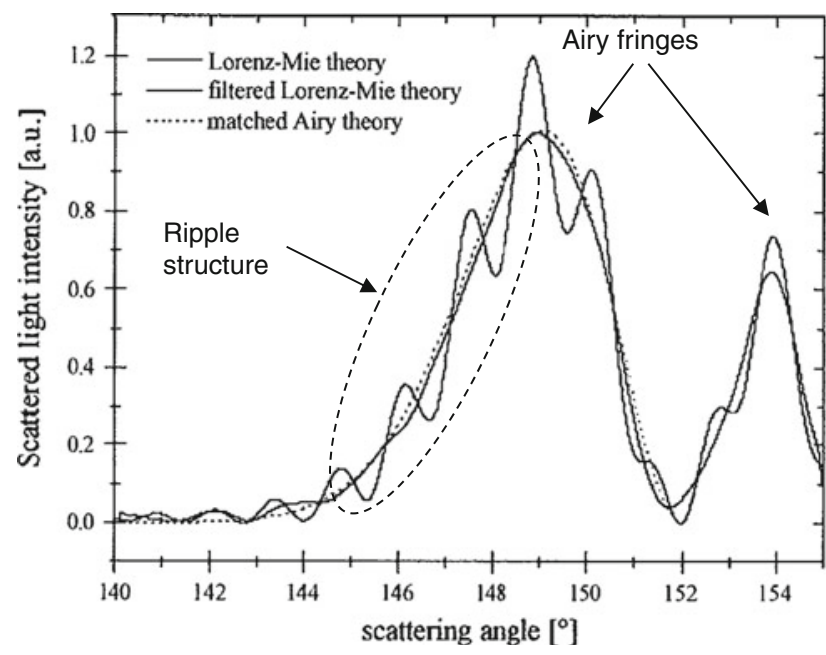

Fig. 4 Scattering pattern by a spherical particle in the vicinity of the primary rainbow computed using LMT. Filtered LMT and Airy theory are also superimposed. Reprinted from Wilms et al. (2004)

have different impact points on the droplet surface. Furthermore, interference between orders 0 and 2 rays give rise to the ripple structure-i.e., a high-frequency modulation of intensity-which Airy's theory does not account for although the LMT does (Fig. 4).

Several strategies have been reported for the inversion of the rainbow signal in SRR. In a first approach demonstrated by van Beeck and Riethmuller (1994, 1995), the droplet size can be determined from the spacing between the Airy fringes without a priori knowledge of the refractive index. The refractive index is then derived from the angular position of the first maximum of the Airy fringes using Eq. (2). Another signal processing solution consists of inferring the droplet diameter from the ripple frequency, and this method may substantially improve the accuracy of the measurements (van Beeck and Riethmuller 1996). Alternatively, Wilms and Weigand (2006) suggested that the refractive index could be determined from the angle of the inflection points of the main peak of the first rainbow instead of the maximum. One noticeable feature of this angle is that it does not depend on the droplet size which may be an advantage in some experiments. As underlined by Damaschke (2003), ripples are a potential source of error in the previous methods of inversion. Results are very sensitive to the location and amplitude of the ripples even though the signal is low pass filtered when compared to Airy's theory. To reduce the uncertainties caused by the ripple structure and the noise, Wilms et al. (2004) used a discrete Fourier transform of the rainbow signal and the Airy theory (van Beeck 1997; Walker 1976).

Inversion algorithms have greatly benefited from recent developments of light scattering calculators based on the Lorentz-Mie theory (LMT) or Generalized Lorentz-Mie theory (GLMT) for focused laser beams (Gouesbet and Gréhan 2011). Both Nussenszweig's complex angular momentum theory (Nussenzveig 1969; Saengkaew et al. 2006) or the Debye-series which enables the separation of the contributions of different scattering orders (Gouesbet and Gréhan 2011) permit fast and accurate calculations. An example of intensity profile computed by LMT in the region of the primary rainbow is presented in Fig. 4. The ripple structure and the Airy fringes are clearly visible in this profile. Figure 4 also shows that the exact solution given by LMT can be filtered to compare with the Airy theory. The possibility of fast calculations with today's computer resources has considerably renewed inversion schemes and led to a dramatic reduction in uncertainties linked with signal processing.

Scattered light is recorded simultaneously in the forward and backward directions. The inversion consists of looking for the best correlation in the Fourier domain (position of the characteristic frequencies and phases) between experimental light scattered profiles and refined calculations based on an exact method like LMT which also take the optical devices' field of view into account (Han et al. 1998; Min and Gomez 1996). Saengkaew et al. (2007) employed this kind of inversion method on a monodisperse stream of ethanol droplets (initial diameter around $95 \mu \mathrm{m}$ ) slowly evaporating in air at ambient temperature. They claimed to have observed a size variation of $0.2 \mu \mathrm{m}$ over $2 \mathrm{~cm}$ and an associated temperature variation of $5{ }^{\circ} \mathrm{C}$. Saengkaew et al. (2010) pointed out the additional benefit in taking into account the ripple structure in the signal inversion.

One noticeable feature of the SRR method is its high sensitivity. For example, a temperature rise from 0 to 
$100{ }^{\circ} \mathrm{C}$ causes a variation of $2.3^{\circ}$ in the rainbow angle of water droplets. However, the deformation of the droplets remains a particular issue of concern. If the droplets are not sufficiently spherical, subsequent distortions of the rainbow pattern may create some ambiguity in the measurement of the refractive index (Han et al. 2002). This kind of error caused by non-sphericity can be avoided in a spray using the global rainbow thermometry described in the next section.

When transient heating and evaporation are under study, gradients of refractive index can arise from non-uniform temperature and composition inside the droplets and size reduction and may significantly affect the measurements (Anders et al. 1996; Massoli 1997, 1998). To test the reliability of the SRR method, Massoli (1998) focused on the critical case of droplets in a reacting flow and carried out numerical simulations based on LMT. In this study, the strong evaporation and heating of the droplets were studied by working with a reduction in the droplet diameter and a radial distribution of refractive index. Results showed a significant decrease in the accuracy of rainbow refractometry. Saengkaew et al. (2007) studied the sensitivity of the SRR to a radial gradient of refractive index and demonstrated that it is always possible to find an equivalent particle with a homogeneous refractive index which scatters light in both forward and backward directions in the same way as a particle with a gradient. However, the temperature corresponding to the refractive index of this equivalent particle is rather difficult to interpret physically.

In the presence of refractive index gradients, measurements by SRR can be misleading (Anders et al. 1996; Schneider and Hilerman 1993). In some cases, measurements may unrealistically indicate cooling, while the droplet is in fact heating because of a higher temperature at the droplet surface than within its interior. The effect of a gradient of refractive index is illustrated in Fig. 5. The higher refractive index at the droplet surface is responsible for a decrease in the scattering angle of the first-order rays and an increase in the angle of the primary rainbow when compared to the homogeneous droplet with an average refractive index. It is clear that the angle of the primary rainbow can be misinterpreted in the case considered in Fig. 5.

The most frequent applications of SRR involve determining the droplet temperature from the refractive index, but the technique can also be used to characterize the chemical composition of droplets in which chemical transformations and reactions are embedded. Wilms et al. (2004) investigated the composition change of evaporating droplets made of n-alkanes mixtures. In these experiments, droplets were optically trapped in a laser beam which allows observations during their complete lifetime. Given the short duration of droplet heating (in relation to their evaporation time), it can be safely assumed that the refractive index depends on the droplet composition exclusively. Figure 6 shows typical results obtained in the case of $n$-hexadecane/n-dodecane droplets. The fraction of $\mathrm{n}$-dodecane within the droplet decreases because n-dodecane is the most volatile compound in the mixture. It is important to note that such measurements are feasible because the refractive index of n-alkanes varies monotonically with the number of carbon atoms. This noticeable feature of n-alkanes was also exploited by Wilms and Weigand (2006) who studied free falling droplets made of n-heptane and n-hexadecane. In this particular case, it was possible to detect the presence of composition gradients inside the droplet thanks to the high difference in volatility of n-heptane and n-hexadecane and so this paper went on to discuss the relevance of models such as the rapid-mixing and the diffusion-limited models for the description of the mechanism of molecular transport inside the droplets (Fig. 6).

More refined utilizations of SRR concerned its coupling with other techniques such as phase Doppler analysis (PDA) and laser-induced fluorescence (LIF). Zhao and Qiu (2006) combined SRR and PDA to study the evaporation of monosized droplets made of ethanol and water. In this study, the refractive index of the liquid mixture was modeled by the Lorentz-Lorentz law, but its determination by SRR requires the knowledge of the droplet diameter.
Fig. 5 Interaction of a light ray with a spherical droplet according to geometrical optics, with and without radial refractive index gradient
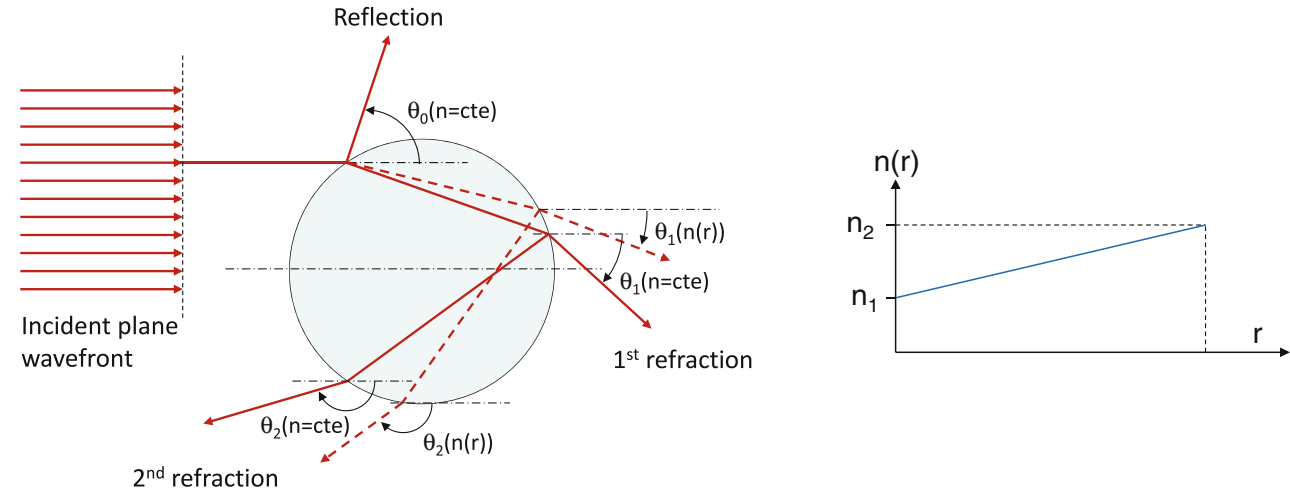


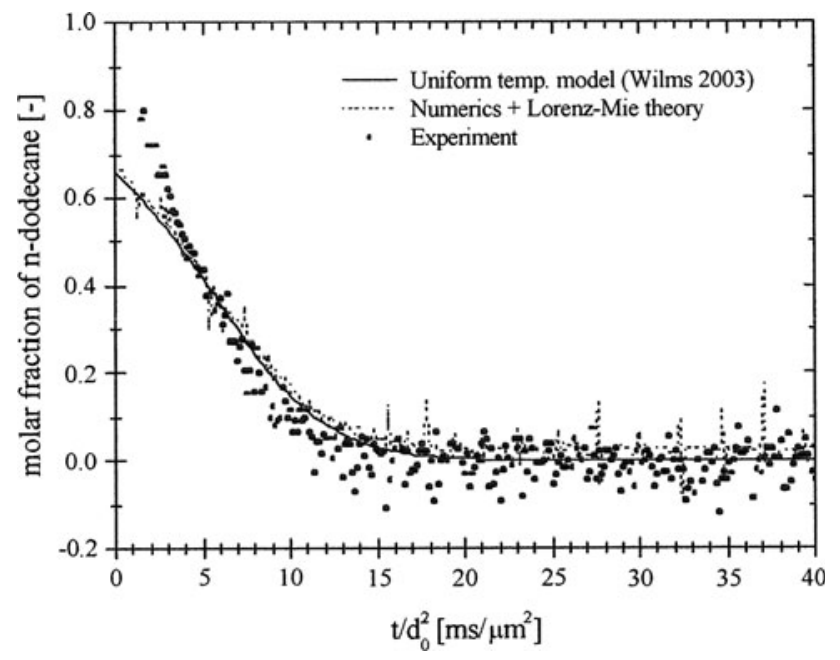

Fig. 6 Evaporation of a binary droplet made of n-hexadecane and $\mathrm{n}$-dodecane: temporal evolution of the $\mathrm{n}$-dodecane molar fraction derived from the refractive index measurement (initial molar fraction of n-dodecane: $66 \%$, initial diameter: $52 \mu \mathrm{m}$ ). Reprinted from Wilms et al. (2004)

The PDA provides an accurate measurement of droplet diameter if the particle refractive index is known but with a rather limited sensitivity to this parameter. However, SRR can be used to measure the refractive index if the droplet size is known. An iterative procedure is thus employed to obtain both the droplet diameter and the refractive index. As the refractive index is a function of both composition and temperature, there is an ambiguity in the interpretation of the measured variations of the refractive index. However, since water evaporation is very weak in comparison with ethanol, Zhao and Qiu (2006) made the assumption that the size reduction in the droplet results from the loss of ethanol exclusively. If all droplets are of the same size at the moment of injection, droplet composition can be deduced from the measurement of the size and thus the respective contributions of temperature and composition to the refractive index measurements can be distinguished. Obviously, this method cannot be applied to the general case of two co-evaporating components because in this case, the composition of the droplet would not be related to the transient droplet diameter.

As already mentioned, inversion algorithms in SRR are capable of handling gradients of refractive index within the droplet thus providing an equivalent particle of homogeneous refractive index with the same scattering pattern as the real particle (Saengkaew et al. 2007). The combination of SRR with another temperature measurement technique is thus of particular interest as it can provide additional information about the temperature distribution within the droplet. Laurent et al. (2006) measured the temperature of evaporating droplets using both SRR and the two-color laser-induced fluorescence technique described in Sect. 3.3.
In this study, the authors assumed a parabolic distribution of the temperature within the droplet. Hence, knowledge of the temperature at the droplet center and the surface temperature suffices to fully characterize the whole temperature profile. Preliminary calculations using Nussenzveig's complex angular momentum theory (McGlashen et al. 1990; Nussenzveig 1969) are performed based on the assumption that the droplet consists of several concentric layers of constant refractive index. These calculations show how the refractive index measured by SRR (refractive index of the uniform equivalent droplet) is related to the refractive index at the droplet center and surface, while 2cLIF measurements provide a volume-averaged temperature which can be also expressed as a combination of both surface and center temperatures provided that the temperature has a parabolic distribution. The combination of both measurement techniques can be used to obtain a set of two equations with two unknown parameters - the center and surface temperatures. The resolution of these equations gives an estimate of the temperature distribution within the droplets.

\subsubsection{Global rainbow refractometry (GRR)}

The GRR technique was initiated by van Beeck et al. (1999) and can be seen as an extension of SRR to sprays. Potentially, GRR is capable of providing valuable information about the size distribution and the temperature associated with an ensemble of droplets. Furthermore, the aim of this method is to eliminate some of the biases of SRR, especially those related to the presence of deformed droplets because non-spherical droplets are a major source of errors. A sphericity default of only $1 \%$ may lead to errors in temperature measurements as high as $\pm 40{ }^{\circ} \mathrm{C}$ (Moebius 1910; van Beeck et al. 1999). van Beeck et al. (1999) attempted to select spherical droplets for processing according to the comparison of droplet size measurements from the Airy fringes and from the ripple.

The optical setup of GRR is very similar to that of SRR, the main difference being the suppression of the spatial filter to select single drops in the probe volume. An extended laser beam is required to simultaneously illuminate numerous droplets and a CCD camera takes images of the global rainbow. It is important to note that the GRR technique works with droplets of different sizes and shapes. The global rainbow results from the superimposition of the rainbows produced by the illuminated droplets. Since spherical droplets have approximately the same refractive index in the probe volume, the angular position of their rainbows is identical and the global rainbow is therefore mainly formed by the constructive interferences of the rainbows of the spherical droplets. Conversely, nonspherical objects like spheroids or ligaments cause 
destructive interferences. A uniform background will result from the summation of their signals provided that the nonspherical objects are randomly oriented. It is commonly accepted that about 1,000 droplets in the probe volume are required to create a converged pattern of global rainbow. In practice, this implies that the attainable probe volume size in GRR depends on spray density. A typical image of a global rainbow recorded in an isothermal water spray is presented in Fig. 7a, while its corresponding intensity profile is displayed in Fig. 7b. In Fig. 7, the Airy fringes are clearly distinguishable due to the absence of ripple. The disappearance of the ripple structure is an interesting feature of the GRR method since ripples had generally been viewed as an obstacle for accurately locating the rainbow maximum in SRR (Roth et al. 1992).

Concerning the inversion of the measurements, van Beeck et al. (2001) demonstrated that the temperature can be derived from the position of the two inflexion points located around the principal rainbow maximum. The reliability of this method was assessed by simulations of the global rainbow based on LMT. If the accurate droplet size distribution is known, uncertainty can be mainly attributed to the difficulty in locating inflexion points with temperature uncertainties only involving a few ${ }^{\circ} \mathrm{C}$. The inversion procedure using the angular position of the inflexion points was successfully applied to the characterization of an isothermal water spray (van Beeck et al. 2003). In the case of water sprays, errors do not exceed $4{ }^{\circ} \mathrm{C}$ according to different studies (van Beeck et al. 1999, 2001). In general, the mean droplet diameter derived from the angular distance of the inflection points around the first rainbow maxima greatly differs from the Sauter mean diameter, but an estimate of size dispersion can still be obtained (van Beeck et al. 2001). Vetrano et al. (2004) highlighted the fact that the previous inversion method has some inherent weaknesses as it is based on characteristic points of rainbowscattered light distribution. To address this issue, they developed a more robust inversion algorithm based on the least square fit of the whole rainbow pattern. The method was demonstrated on silicon oil droplets suspended in a water bulk with a quite large distribution of droplet sizes.

Based on the preliminary works of Han et al. (2002) and Bonin et al. (2007), Wilms et al. (2008) demonstrated that the contribution of non-spherical droplets to the global rainbow is not in fact a uniform background. They found that measurements of droplet size and refractive index are not affected to the same extent when making this erroneous assumption. A variation of the GRR method was proposed by Wilms et al. (2008) who aimed to eliminate the negative contribution of non-spherical droplets. In this approach, images of rainbows derived from single droplets were recorded in the spray using an appropriate spatial filter. A global rainbow was then reconstructed by superimposing the images of the rainbows from single droplets after elimination of the images corresponding to non-spherical drops. Several criteria were used to filter the non-spherical droplets: the intensity ratio of the first two maxima, the ratio of the angular spacing of the first two minima and maxima of the rainbow. The method was successfully tested in the vicinity of the outlet orifice of an atomizer where a large amount of droplets are non-spherical. The droplet size distribution was compared to results obtained using a PDA, and a better success rate was found using these new criteria of sphericity than with the traditional inversion algorithm.

Using the same method for the image acquisition, Saengkaew et al. (2009) analyzed the effect of a proportion of non-spherical droplets on the temperature and size distribution measurements by using time series images of nonspherical droplets (ellipticity varying from 0.988 to 1.012). After application of the sphericity criteria, the temperature was measured with an accuracy of $2{ }^{\circ} \mathrm{C}$ and the obtained droplet size distribution was found to comply well good agreement with data obtained with a PDA. Moreover, the
Fig. 7 Illustration of the global rainbow. a Global rainbow pattern obtained in a water spray, b profiles of global rainbow pattern (scattering angle is proportional to the pixel number). The different plots correspond to different instants. $\theta_{1}$ and $\theta_{2}$ are the angular position of the two Airy first maxima. Reprinted from van Beeck et al. (2003) (a)

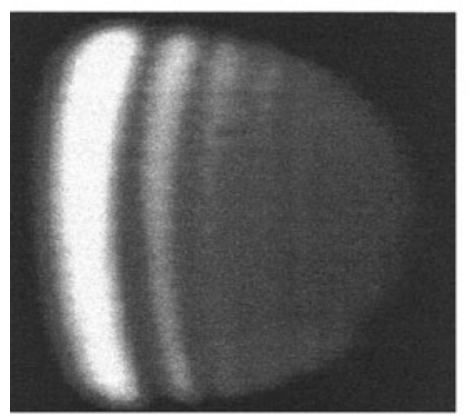

(b)

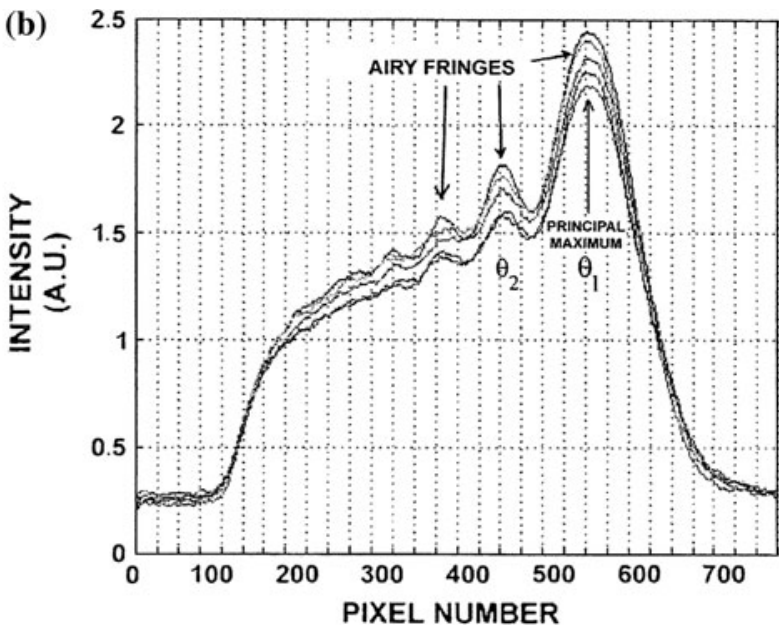


temperature accuracy was only about $5{ }^{\circ} \mathrm{C}$, and the droplet size distribution was strongly affected by small ghost particles, without application of the sphericity criteria.

Saengkaew et al. (2012) proposed combining GRR and PDA with the aim of obtaining refractive index by class of droplet size in the sprays. They supposed that droplet size distribution had been correctly obtained using PDA measurements and went on to develop a two-step inversion algorithm. The main drawback of the method is that every droplet within a class of size is assumed to have the same refractive index. Furthermore, the probe volume dimensions of the PDA and global rainbow may be slightly different which is also a potential source of error. The method was applied to monitor the capture of $\mathrm{CO}_{2}$ by monoethanolamine droplets. In this application, the dependence of the refractive index on both composition and temperature is not a problem because the effect of the composition is dominant in the conditions of the experiments.

\subsection{Techniques based on Raman scattering spectroscopy}

Raman scattering was widely used to characterize the chemical composition and to some extent the temperature of droplets. In particular, spontaneous Raman scattering provides indirect measurements of chemical composition, size and temperature. The method is also able to distinguish between liquid and vapor and to monitor phase changes in the droplet such as crystallization during the droplet drying for example. Cavity-enhanced Raman scattering allows a significant amplification of the signal. This approach has mainly been used to probe composition changes near the surface of droplets.

\subsubsection{General concepts}

Raman scattering results from non-elastic collisions of photons on nuclei and electron clouds of the molecules (Schweiger 1990). The net exchange of energy between photons and molecules may result in an increase or a decrease in the molecules' vibrational or rotational energies. Because of this process of energy conversion, the scattered Raman photons are blue- or red-shifted, i.e., they contain more or less energy as compared with incident photons. A stokes line (of energy $h\left(\omega_{0}-\omega_{\mathrm{k}}\right.$ ), where $\omega_{0}$ is the incident photons frequency and $\omega_{\mathrm{k}}$ is the frequency difference due to the Raman shift) and an anti-stokes line (of energy $h\left(\omega_{0}+\omega_{\mathrm{k}}\right)$ ) can be found in the Raman spectrum, where they form a symmetric pattern above and below the incident photon energy. The frequency shift associated with the Raman effect depends on the nature of the scattering molecules, while the intensity of the Raman scattering is a function of the number density of the molecules and to some extent the temperature through the Boltzmann fraction (Tropea et al. 2007). The intensity of the Raman scattering is so weak that powerful and highly focused laser sources are usually necessary for the measurements. To give an idea, the Raman scattering crosssection of common molecules is about $10^{-30} \mathrm{~cm}^{2}$ which is ten orders of magnitude smaller than the fluorescence cross-section of the molecules used in most of LIF's applications. The Raman signal consists of photons collected from a specific probe volume where molecules of the chemical specie $c$ are excited at a specific light frequency $\omega_{0}$. The Raman scattering flux $S_{c}$ radiated in a solid angle $\Omega_{0}$ can be expressed as:

$S_{c} \sim \frac{1}{\hbar \omega} \frac{\partial \sigma^{c}}{\partial \Omega} I_{0} N_{c} \Omega_{0}$,

where $\omega=\omega_{0} \pm \omega_{\mathrm{k}}$ is the Raman scattering frequency, $N_{c}$ is the number of $c$ molecules in the probe volume, $I_{0}$ is the incident local excitation intensity and $\hbar=h / 2 \pi, h$ being the Planck constant. The Raman scattering cross-section of the chemical specie $c$, denoted $\sigma^{c}$, is temperature dependent through the Boltzmann fraction, i.e., the fraction of $c$ molecules occupying a given vibrational and rotational mode at the considered temperature. The thermal dependence of Raman scattering has largely been used for thermometry in gases at high temperature. However, in some liquids, the influence of hydrogen bonds on the Raman shift becomes dominant and thermally dependent. More details about Raman scattering can be found in Anderson (1971, 1973) and Herzberg (1945, 1950).

With Raman scattering from droplets, the Raman signal arises from active molecules embedded in the droplet interacting with the incident excitation field. In this context, two processes should be considered to describe the Raman emission from the droplet. The first is the establishment of the field within the droplet at the incident laser wavelength, while the second is the establishment of the Raman field shifted in wavelength. In a simplified approach to describe the Raman scattering by a particle, the intensity of the Raman emission from the molecules inside the particle is considered to be proportional to the local intensity of the excitation field, although resonances can also take place. Resonances associated with the elastic field or whispering gallery modes (WGMs) will also correspond to resonances in Raman intensity (input resonances), but resonances associated with the Raman field (which is wavelength shifted) will lead to stimulated Raman emission (output resonance).

To describe the Raman scattering from particles, Eckbreth et al. (1979) introduced the derivative of the Raman cross-section per solid angle and scattering wavelength, $\partial^{2} \sigma^{c} / \partial \Omega \partial \lambda_{s}$. The radiant flux $W_{c}$ emitted from a single 
particle where the specie $c$ is homogeneously distributed is given by:

$W_{c}=I_{0} \frac{1}{\hbar \omega} \frac{N_{a v} \rho_{c} V_{d}}{M_{c}} \int_{\Delta \lambda_{s}} \int_{\Omega_{0}}\left(\frac{\partial^{2} \sigma^{c}}{\partial \Omega \partial \lambda_{s}}\right) d \Omega d \lambda_{s}$,

where $V_{d}$ is the volume of the particle, $\rho_{c}$ is the specific density of the specie $c, M_{c}$ its molar mass, $N_{a v}$ is the Avogadro number, $\Omega_{0}$ is the considered solid angle of the emission and $\Delta \lambda_{\mathrm{s}}$ is the spectral band over which the Raman photons are radiated.

\subsubsection{Spontaneous Raman scattering}

The main applications of spontaneous Raman scattering to droplets concern the characterization of their in situ chemical composition. Temperature measurements are also achievable but less common. As shown in Fig. 8, a typical Raman scattering setup includes a highly focused laser which can be operated in continuous or pulsed modes and a CCD camera coupled with a spectrograph. When a pulsed excitation is used for the investigation of periodically flowing droplets-as seen in Fig. 8-synchronization is required between the laser pulses and droplets. The principles behind the main applications of Raman scattering to droplets were reviewed by Schweiger (1990). Schweiger (1987) and Davis et al. (1990) defined the basic concepts of Raman scattering applied to the in situ analysis of the composition of aerosols. Certain difficulties in analyzing the molecular composition of droplets can be associated with the effects of droplet size and shape because the droplet interface focuses the incident laser light inside the droplet. Since the Raman signal is proportional to the local excitation power density, the lensing effect produced by the droplet interface results in an inhomogeneous distribution of the sources of Raman scattering within the droplet and a non-uniform angular distribution of the Raman signal scattered by the droplet.

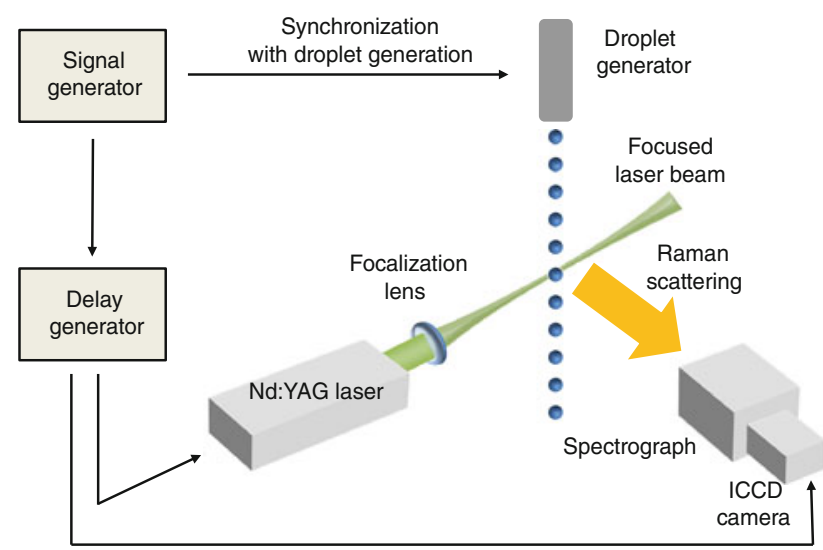

Fig. 8 Typical Raman spectroscopy setup
Additionally, morphology-dependent resonances (MDRs) also called whispering gallery modes (WGMs) (Hill and Brenner 1988) complicate the interpretation of the Raman spectrum by adding peaks corresponding to Raman frequencies which fulfill the resonance conditions (Owen et al. 1982). This phenomenon can be exploited in techniques based on stimulated Raman scattering spectroscopy which are detailed in the next section. Resonance frequencies are highly sensitive to the size and the refractive index of the droplets and thus they are changing during the evaporation and heating of the droplets or when a chemical transformation is embedded in the droplets.

The use of monosized droplets,- - since they all have the same diameter and refractive index at a designated location-tends to enhance the consequences of the already mentioned MDR peaks, but space and time averaging of the signal is generally used to smooth the resonances (Vehring et al. 1995).

In a pioneer work on Raman scattering applied to droplets, Carls et al. (1990) investigated the chemical absorption of $\mathrm{D}_{2} \mathrm{O}$ vapor by an acoustically levitated droplet of glycerol (diameter $25 \mu \mathrm{m}$ ). Although the chemical evolution of the droplet could be clearly observed on the Raman spectra, quantitative interpretation remained difficult due to MDR peaks. Buehler et al. (1991) investigated the evaporation of multicomponent droplets using Raman scattering spectroscopy. They highlighted the difficulties linked to droplets evaporating and undergoing a chemical transformation. In particular, the method requires too large integration time due to the weak Raman signal. Ideally, the integration time of the technique should be much smaller than the time scale of the droplet changes in diameter, refractive index and chemical composition. Vehring et al. (1995) investigated the $\mathrm{CO}_{2}$ desorption from water droplets. In this study, the shift between the peaks corresponding to gaseous $\mathrm{CO}_{2}$ and dissolved $\mathrm{CO}_{2}$ is used to separate the liquid and gaseous phases, while the area of the Raman peaks allows measuring concentrations in both phases. Using this approach, the authors were able to measure profiles of concentration in the gas phase in the close vicinity of the droplet. Vehring et al. (1998) performed similar measurements but extended the method to particles as small as $3 \mu \mathrm{m}$. Stowers and Friedlander (2002) applied Raman spectroscopy to their study on mixing two aerosols flowing into nitrogen with one made of diethylsebacate (DES) and the other of dried ammonium sulfate particles. They performed in situ measurements of the chemical composition of the polydisperse droplets (diameter ranging from 0.3 to $1.8 \mu \mathrm{m}$ ). In this work, numerical simulation was used to demonstrate that the intensity of the Raman signal is roughly proportional to the mass of active material when the size parameters range from 2 to 8 . For the largest size parameters, calculations also showed that 
the fluctuations of the intensity of the Raman signal with the scattering angle were considerably damped compared to the elastic light scattering.

In the experiments, the integration of the Raman signal from many particles allows the oscillations of the signal to be reduced as compared with single particles. Finally, measurements of the chemical composition of the aerosol were achieved in an extended volume with a good level of accuracy by calculating the ratio of the height of the peak associated with nitrogen (the carrier gas) and the height of the peaks corresponding to DES or ammonium sulfate.

Spontaneous Raman scattering was also used to measure droplet temperature. Temperature measurements in liquids are based on the fact that some Raman lines are dependent on intermolecular forces, particularly hydrogen bonds whose strength is affected by the temperature. For instance, in liquid water, the number of hydrogen bonds formed by a molecule of liquid water decreases with the temperature. On that basis, measuring the temperature of liquid water can be performed by using the spectral shape of the $\mathrm{OH}$ stretching band (Vehring and Schweiger 1992; Walrafen 1967). Müller et al. (2000b) used the shape of the $\mathrm{OH}$ stretching band to determine the temperature of ethanol and methanol droplets in a pre-heated direct injection gasoline spray injected into a 50-bar back-pressure chamber. Calibration of the $\mathrm{OH}$ stretching in bulk liquid is required to determine the position of the Raman peak at a given temperature, as illustrated in Fig. 9 which also shows the Raman emission corresponding to the $\mathrm{OH}$ emission of the vapor phase. This emission is shifted toward shorter wavelengths compared to the liquid phase. According to Müller et al. (2000b), the technique has a high level of accuracy, typically of around $\pm 1{ }^{\circ} \mathrm{C}$. These temperature measurements were spatially resolved but not timeresolved due to long acquisition time of the Raman spectra.

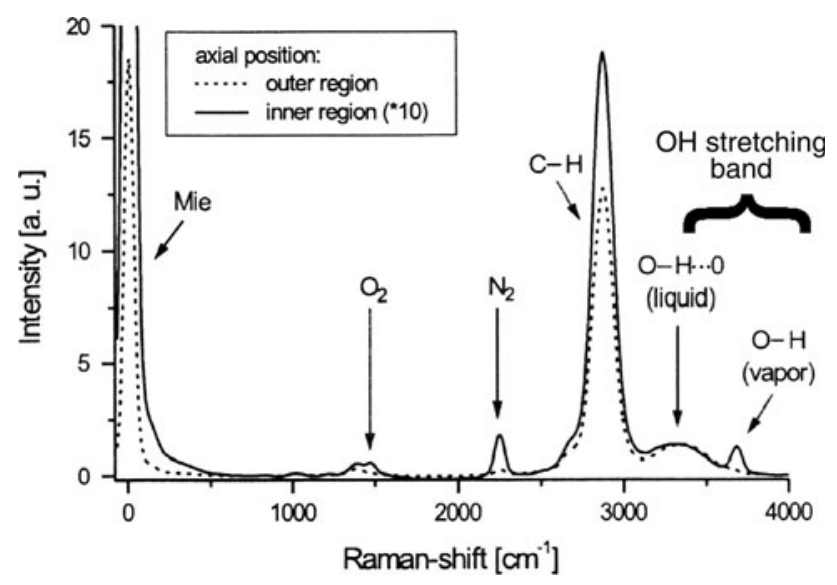

Fig. 9 Typical Raman spectrum recorded in a spray inner region corresponds to the spray core. The outer region corresponds to the sprays edge. Reprinted from Müller et al. (2000b)
Another issue relates to multiple scattering of the incident laser radiation which can modify the polarization of the incident laser excitation in dense sprays. This is a major problem since the temperature sensitivity of some Raman peaks is affected by the polarization direction of incident laser light. Müller et al. (2000a) carried out a 2D characterization of an ethanol and methanol pulsed spray. In this study, spatially resolved measurements of liquid and vapor concentrations, droplet temperatures and sizes were achieved. The spectral shift between the Raman emission of the liquid and vapor phases was used to discriminate between liquid and gas phases. The overall magnitude of the Raman peaks in the spectral bands corresponding to the liquid and the gas are compared which meant that the average fractions of liquid and vapor in the spray could be determined. The Sauter mean diameter of the droplets was derived from the ratio of Raman scattering (roughly proportional to the droplet volume $D^{3}$ ) and Mie scattering (roughly proportional to droplet surface area $D^{2}$ ). Heinisch et al. (2009) investigated the evaporation of water droplets levitated in an electrodynamic trap where nitrogen was injected at different velocities. Here also, the $\mathrm{OH}$ stretching band was used to measure droplet temperature after a calibration. A noticeable improvement is the use of a ratio between the signals collected on two spectral windows, one located in the vicinity of the maximum of the $\mathrm{OH}$ stretching band and the other at higher frequency where the spectrum is the most sensitive to the temperature. The thermal dependence of this ratio is about $0.45{ }^{\circ} \mathrm{C} / \%$, while the temporal resolution is on the order of $0.3 \mathrm{~s}$ and the overall accuracy $\pm 4{ }^{\circ} \mathrm{C}$.

\subsubsection{Stimulated Raman scattering spectroscopy}

Stimulated Raman can be exploited to characterize the composition of multicomponent droplets, in particular ethanol-water droplets (Homer et al. 2009; Hopkins and Reid 2005; Hopkins et al. 2003; Howle et al. 2007; Reid et al. 2007). The technique is called cavity-enhanced Raman scattering (CERS) and was initiated by Hopkins et al. (2003). It is also referred to as surface-enhanced Raman scattering (SERS) in the particular case of droplets. For some particular wavelengths, light waves called whispering gallery modes (WGMs) can be guided around a droplet almost perfectly by total optical internal reflection. As illustrated in Fig. 10, light in WGMs is trapped in the close vicinity of the droplet surface. Amplification of light occurs in a WGM, as the droplet acts as a low-loss optical resonant micro-cavity. This noticeable feature is exploited in CERS to produce stimulated Raman scattering in a droplet at wavelengths of the Raman scattering emission that is commensurate with WGMs (Biswas et al. 1989; Lin et al. 1990; Reid et al. 2007). Furthermore, when incident 


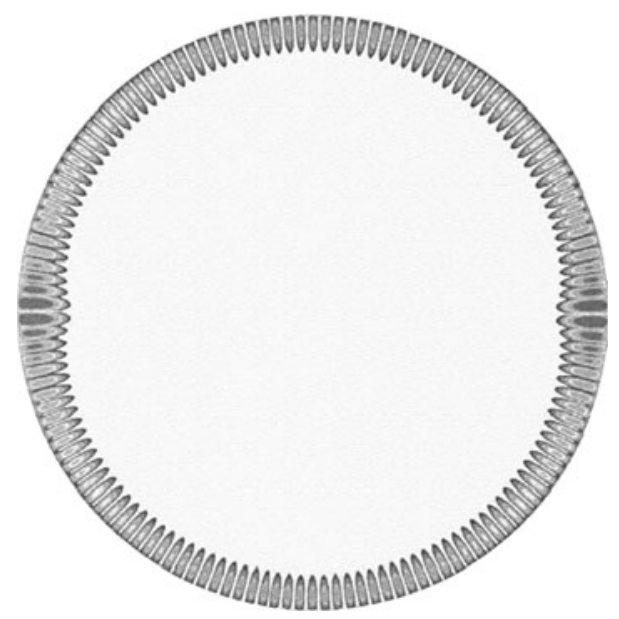

Fig. 10 Illustration of the intensity field of a whispering gallery mode (WGM). Reprinted from Reid et al. (2007)

laser light is also trapped within a WGM, double-resonance stimulated Raman scattering may occur. This phenomenon is characterized by very high gains in amplification. The obtained Raman spectrum has a structure which depends on droplet size and WGMs wavelengths. Therefore, it can be used to determine droplet size very accurately when recorded on a single droplet. However, to determine droplet composition, a spectrum composed of spectra recorded on hundreds of droplets which are naturally only slightly different in size is required to smooth the sizedependent resonances. Alternatively, the droplets can be gently modulated in size to obtain a smooth spectrum resulting from the summation of several single shot spectra which include characteristic spectral peaks, corresponding to individual droplets.

Cavity-enhanced Raman scattering (CERS) spectral bands exhibit considerable narrowing compared to bulk measurements due to the high gain at the wavelength of the maximum of the Raman band due to light amplification at particular wavelengths in WGMs. A noticeable feature of CERS is that signal is exponentially sensitive to the droplet composition under unsaturated conditions, i.e., when the signal remains proportional to the number of scatterers (4) (Reid et al. 2007):

$I(C E R S, \lambda)=S(\lambda) e^{(g S(\lambda))}$

where $I(\mathrm{CERS}, \lambda)$ is the CERS signal at wavelength $\lambda, S(\lambda)$ is the spontaneous Raman scattering intensity at the same wavelength and $g$ is the cavity gain factor (Hopkins et al. 2003). This gain is proportional to the molecular number concentration of scatterers and the intensity of incident light trapped into the WGM.

The technique was successfully used in the study of ethanol-water droplets falling at ambient temperature and pressure in air by stimulating both $\mathrm{CH}$ and $\mathrm{OH}$ stretching

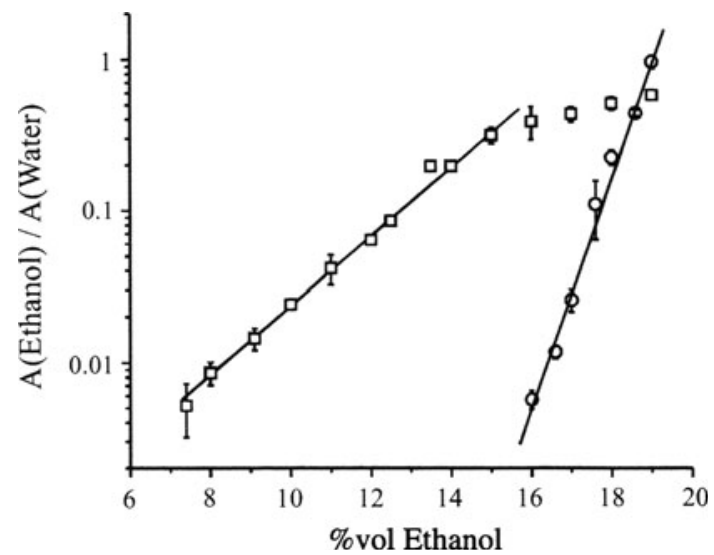

Fig. 11 Example of calibration curve of the ethanol CERS signal as a function of composition. A is the integrated intensity on the spectral band (squares excitation at $532 \mathrm{~nm}$, circles excitation at $355 \mathrm{~nm}$ ). Reprinted from Hopkins et al. (2003)

bands for ethanol and water, respectively. The ratio between the integrated intensity of CERS signal coming from water and ethanol was found to decease by half, while the ethanol volume fraction decreased by only $0.6 \%$ (Hopkins et al. 2003). This provided highly accurate composition measurements of the composition change (Fig. 11). It should be noted that the sensitivity of this ratio also depends on the excitation wavelength of the laser.

More sophisticated experiments and analysis can be found in Hopkins and Reid (2005). In this work, a train of monodisperse ethanol-water droplets (diameter range: $25-57 \mu \mathrm{m}$ ) was introduced into a dry nitrogen atmosphere at a pressure ranging from 7 to $77 \mathrm{kPa}$. The authors underline the fact that light trapped in WGMs only propagates in a depth of about $r / m$ ( $r$ being the droplet radius and $m$ the real part of the refractive index of the liquid) from the droplet surface. Consequently, the CERS signal only comes from a layer located near the droplet surface whose thickness do not scales with the diffusion length of ethanol. Consequently, the change in the composition measured by the technique in the outer layer of the droplet cannot be directly interpreted as the change in composition of the overall droplet and some additional corrections, based on modeling of transport phenomena within the droplet need to be implemented as suggested by Hopkins and Reid (2005).

Hopkins and Reid (2006) and Homer et al. (2009) underlined that the CERS signal was more spatially confined than the penetration depth of the WGM; the resulting probed thickness of the CERS signal was found to be about $3 \%$ of the droplet radius. This means that the timedependent change in the near droplet surface composition of an evaporating bicomponent droplet can be measured. A refined analysis of the chemical composition of acoustically levitated millimeter droplets by using CERS was 
carried out by Tuckermann et al. (2009). In this study, the CERS technique was applied to evaporating methanol droplets as well as droplets containing an aqueous solution of sodium ammonium sulfate $\left(\mathrm{NH}_{4}\right)_{2} \mathrm{SO}_{4}$ solution which crystallizes during the droplet drying. The effect of ambient humidity on the evaporation of methanol droplets was investigated by monitoring the red-shift $\mathrm{CO}$ vibration band. As illustrated in Fig. 12, the intensity of the C-O band decreases by a factor 5 and the red-shift neighbored $17 \mathrm{~cm}^{-1}$ as hydration of the droplets by water condensation occurs. Crystallization in salty droplets was monitored using the $\mathrm{SO}_{4}{ }^{2-}$ band which is red-shifted in the crystalline phase compared to the aqueous phase. However, the interpretation of the Raman spectra presented in Fig. 13 (for both the aqueous saturated solutions and the crystalline phase) remains mainly qualitative.

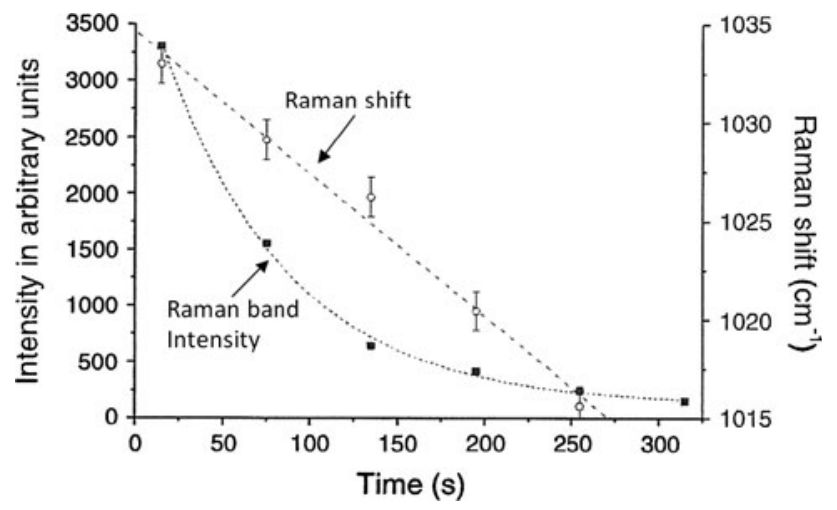

Fig. 12 Evaporation of acoustically levitated methanol droplets in ambient air. Evolution of the Raman band intensity ( $\mathrm{C}-\mathrm{O}$ band) and Raman shift during the evaporation phase. Reprinted from Tuckermann et al. (2009)

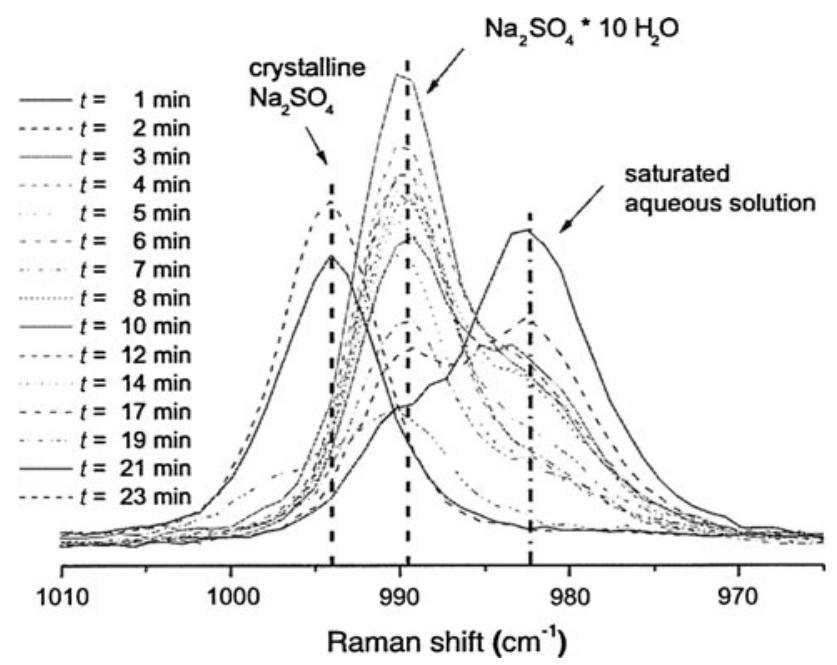

Fig. 13 Raman spectra of an evaporating and crystalizing $\mathrm{Na}_{2} \mathrm{SO}_{4}$ droplet recorded at different instant of the evaporation. Reprinted from Tuckermann et al. (2009)
In Hopkins et al. (2006), the CERS technique was applied to near surface temperature measurements based on the shape of the $\mathrm{OH}$ stretching band. The measurements were only qualitative since a calibration of the CERS signal as a function of the temperature cannot be performed in bulk liquid. In this study, laser-induced fluorescence was also used to determine the volume-averaged temperature of the droplets doped with Rhodamine B.

\section{Measurement techniques based on laser-induced fluorescence}

Laser-induced fluorescence (LIF) is widely used for flow visualization and quantitative measurements. Applications in droplets are mainly related to temperature measurements and, to a lesser extent, to concentration measurements. The use of intensity ratios is almost unavoidable if signal disturbances that may originate from many influences are to be eliminated. If the appropriate intensity ratios are used to determine temperature, these almost totally cancel out the influence of the composition of the liquid.

As an alternative to these intensity-based methods, lifetime-based methods provide a direct access to an intrinsic parameter namely the fluorescence lifetime which is related to the quenching rate of the fluorescence and thus to the temperature and composition of the probed medium. Lifetime methods inherently do not require the same care as intensity-based methods to eliminate any signal disturbances not directly related to temperature or composition.

\subsection{Fluorescence signal of a droplet}

Fluorescence is a form of luminescence which results from the spontaneous emission of a photon by a molecule which has been excited to a quantum state of higher energy corresponding to a singlet state. The frequency of exciting light is different from the frequency of emitted light. In general, fluorescence emissions have a longer wavelength than the absorbed light. This phenomenon is due to energy loss between the time a photon is absorbed and when it is emitted. When the excited molecule returns to its ground state, many pathways can compete with fluorescence including collisional quenching, intersystem crossing, internal conversion, quenching by energy transfer and charge transfer reaction (Lakowicz 2006). All of these relaxation mechanisms contribute to a quenching of the fluorescence emitted by the population of molecules. The fluorescence quantum yield $\phi$ corresponds to the ratio of the total energy emitted per quantum of energy absorbed by the molecules: 


$$
\phi=\frac{A}{A+Q_{1}+Q_{2}},
$$

where $Q_{1}$ is the collisional quenching rate, $Q_{2}$ is the rate of non-radiative decay by any other processes than collisions and $A$ is the fluorescence emission rate. Temperature is likely to modify significantly the different non-radiative decay processes. Fluorescence typically follows first-order kinetics. After an excitation by an ultrashort pulse of light, the fluorescence emitted by the population of molecules will decay with time according to:

$I_{f}=I_{f, 0} e^{\frac{t}{\tau}}$,

where $I_{f}$ is the fluorescence signal intensity at time $t, I_{f, 0}$ is its initial value. The lifetime $\tau$ is independent of the initial intensity of the emitted light which is advantageous for measurements. Similarly with the fluorescence quantum yield, the rate of the relaxation pathways modifies the excited state lifetime. The inverse of the lifetime is the sum of the rates for each decay pathway:

$\frac{1}{\tau}=A+Q_{1}+Q_{2}$.

If light excitation lasts a lot longer than the fluorescence lifetime, fluorescence emission is the convolution of the exponential fluorescence decay in Eq. (7) and the temporal profile of the excitation light source. When the latter extends over a much longer period than the lifetime, the fluorescence signal $I_{f}$ at a wavelength $\lambda$, probed by an optical system in a volume $V_{c}$, i.e., the part of the droplet volume in the field of view of the detector, is given by Lavieille et al. (2001):

$I_{f}(\lambda)=K_{\text {opt }}(\lambda) I_{0} \varepsilon_{0} C \phi V_{c}$,

where $I_{0}$ is the incident light flux $\left(\mathrm{W} / \mathrm{m}^{2}\right), C$ is the concentration of the fluorescent molecules $\left(\mathrm{mol} / \mathrm{m}^{3}\right)$ and $\varepsilon_{0}$ $\left(\mathrm{m}^{2} / \mathrm{mol}\right)$ is the absorption coefficient at the wavelength of the illuminating laser. Equation (9) is valid if the incident light flux is sufficiently small to avoid nonlinear effects such as saturation or photobleaching. According to Lavieille et al. (2001), an acceptable expression for the fluorescent signal emitted by a droplet is:

$I_{f}(\lambda)=K_{\mathrm{opt}}(\lambda) C V_{c} I_{0} \varepsilon_{0} \phi$.

Equation (10) is also worthy of comment. The lens effect provided by the droplet interface is responsible for the focusing of the incident laser beam (Fig. 14). Consequently, the distribution of the laser light flux is not uniform inside the droplet even if the laser beam is assumed to be a plane wave outside the droplet in Eq. (10). For the same reason, the detector cannot view the entire inner volume of the droplet and blind zones remain whatever the observation angle of the detector. The product $C V_{c} I_{0} \varepsilon_{0}$ in Eq. (10) should be regarded as the energy absorbed by the fluorescent molecules in the observed volume of the droplet by unit of time. Even if the ray paths are short in droplets, absorption loss may be significant particularly when the fluorescent molecules are a major component of the droplets rather than a tracer as in Maqua et al. (2007) where acetone was a volatile component of a
Fig. 14 Excitation field within a droplet illuminated by a plane wave coming from the left side. (Acetone volume fraction is $Z_{\mathrm{a}}=20 \%(\mathbf{a})$ and $Z_{\mathrm{a}}=90 \%$ (b). Left calculation by ray tracing; right experiment). Reprinted from Maqua et al. (2007) (a) $Z_{a}=20 \%, m=2.510^{-4}, D=109 \mu \mathrm{m}$
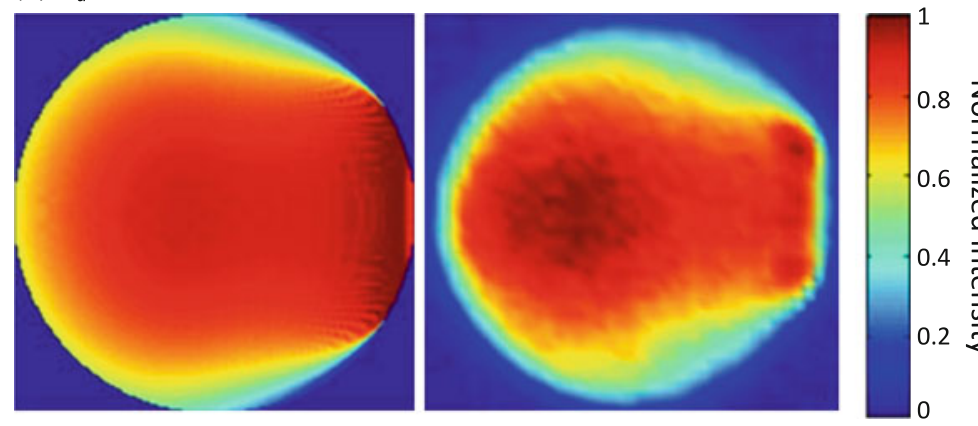

(b) $Z_{a}=90 \%, m=9.310^{-4}, D=187 \mu \mathrm{m}$
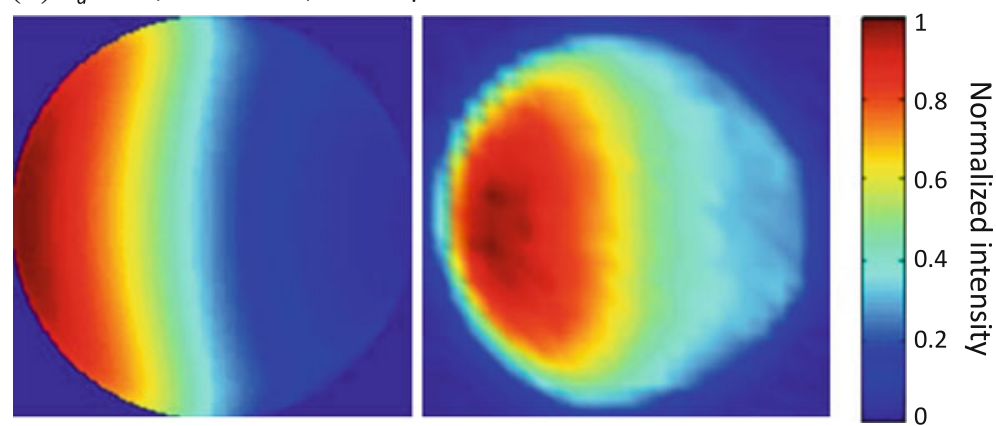
binary mixture used as a fluorescent tracer (Fig. 14). Equation (10) does not account for the effects of the absorption of the laser beam and the reabsorption of fluorescence itself across the absorbing medium. Absorption must be considered through the imaginary part of the refractive index and the Beer's law.

The Mie theory and geometrical optics were used to rigorously calculate the excitation field inside the droplet and the fluorescence signal emerging toward the detector (Castanet et al. 2005a; Domann and Hardalupas 2001; Domann et al. 2002; Frackowiak and Tropea 2010a, b; Zhang and Melton 1993). In the case of a laser beam arbitrary shaped like a Gaussian beam, the Generalized Lorenz-Mie theories (Gouesbet and Gréhan 2011) can be used as an extension of the Mie theory. Geometrical optics was used in association with ray tracing to describe the propagation of light in terms of rays whose paths are governed by Snell's law and whose intensity changes at the interfaces are calculated according to the Fresnel coefficients (Frackowiak and Tropea 2010a). Paraxial approximation and ray transfer matrix analysis have been also used to describe the internal excitation field (Lavieille et al. 2000). Among all the calculations, there is general agreement on the fact that the fluorescence signal from a droplet is not proportional to the droplet volume (Domann et al. 2002; Frackowiak and Tropea 2010a). Due to the lens effect induced by the droplet surface and the absorption losses, the excitation field of fluorescence is not uniform within the droplet which means fluorescence is emitted by different points with different probabilities of reaching the detector. This results in an uneven weighting of the quantities measured by LIF, meaning that LIF measurements rarely correspond to the arithmetic volume average.

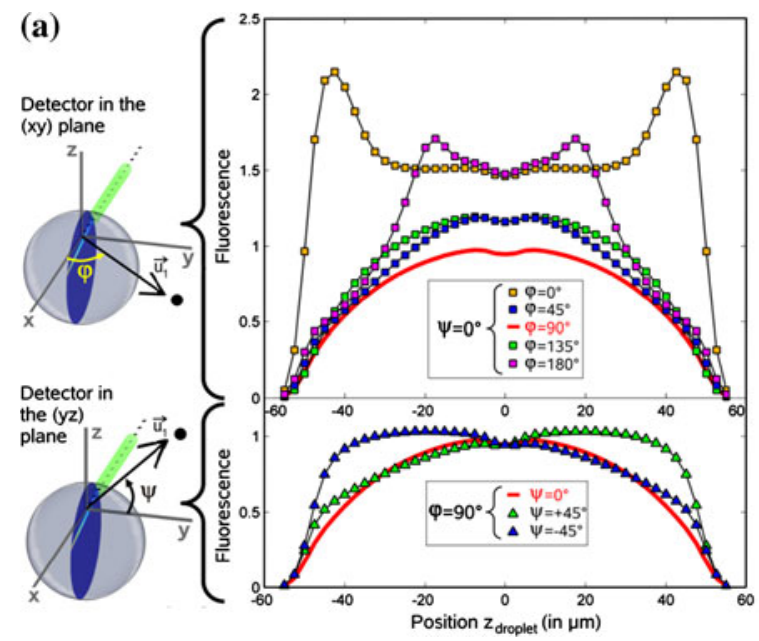

Furthermore, when a droplet crosses the laser beam and the detection volume, the fluorescence signal varies with time. The shape and amplitude of the signal depend on many parameters including the size of the droplet relative to the diameter of the laser beam, the angular position and solid angle of the detection system, the droplet trajectory (if the droplet is off-center relatively to the laser beam and the focal point of the detection optics) and the real and imaginary parts of the refractive index. Some of these dependences are illustrated in Fig. 15 from the work of Frackowiak and Tropea (2010a). Figure 15 clearly shows that intensitybased measurements may be subject to many disturbances and for this reason almost all the measurement techniques based on LIF used in droplets are based either on intensity ratios or on the fluorescent lifetime. The same applies to techniques based on laser-induced phosphorescence.

\subsection{Composition measurements based on the fluorescence intensity}

From a general point of view, the intensity of the fluorescence signal has been used to measure the concentration of fluorescent molecules (seeded or naturally present) in many liquid flows (Crimaldi 2008; Lemoine et al. 1996; Walker 1987). Adapting these methods to droplets are particularly challenging because it requires measuring absolute intensities rather than relative intensities. The fluorescence signal of droplets is a complex function of many parameters which may be difficult to control when attempting to reproduce such measurements. This certainly explains why few papers have dealt with LIF applications for concentration measurements in droplets.

Shringi et al. (2009) visualized the fluorescence emitted by acetone inside individual droplets composed of

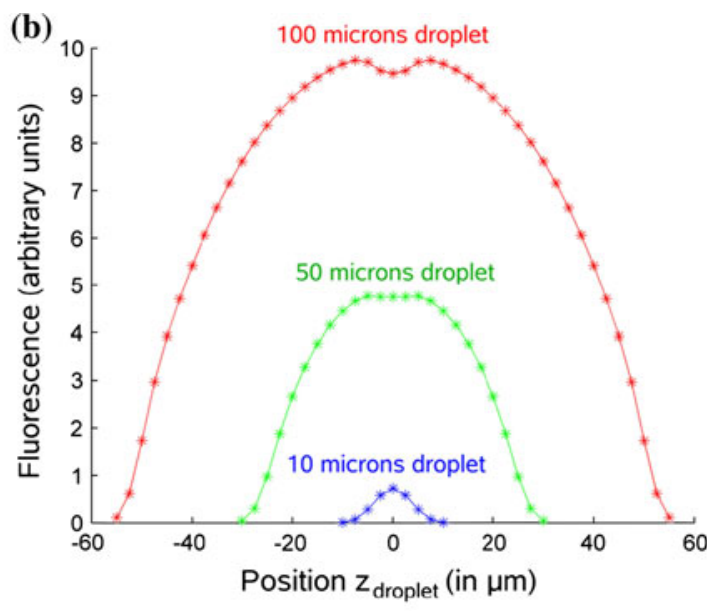

Fig. 15 Variation of the fluorescence intensity of a droplet traversing a focused laser beam $\mathbf{a}$ influence of the detector position, $\mathbf{b}$ influence of the droplet diameter. Reprinted from Frackowiak and Tropea (2010a) 
methanol or 1-propanol initially mixed with acetone. They exploited the fact that acetone absorbs UV light and emits fluorescence partially in the visible spectrum, whereas alcohols like methanol or propanol do not fluoresce. Droplets supported by a wire were illuminated by a pulsed laser at $308 \mathrm{~nm}$. Significant decreases in fluorescence intensities were observed as the droplets evaporated in a flame or in quiescent air at room temperature. Even if the images show many interesting features indicating the presence of internal convection, results remain qualitative since the measured intensities were not converted into acetone fractions.

Maqua et al. (2007) characterized the acetone fraction in droplets composed of binary mixtures made of ethanol and acetone droplets. Measurements were performed on monodisperse droplet. The pulses of a $\mathrm{Nd}$ :Yag laser quadrupled in frequency $(\lambda=266 \mathrm{~nm})$ were synchronized on the droplet transit in the laser beam so that the CCD camera could take images of the droplets when they were perfectly centered in the probe volume. Results show a significant effect of the absorption by acetone on the energy distribution inside the droplet (Fig. 14a). Assuming the concentration within the droplet to be uniform, Maqua et al. (2007) developed an inverse model based on ray tracing (Fig. 14b) to estimate the volume fraction of acetone, taking into account the strong absorption by acetone which modifies the incident excitation field. The method was demonstrated to be quantitative for moderate acetone fraction (typically below $20 \%$ ). As the fraction of acetone increased, fluorescence emission was found to come from a thin region near the droplet surface. The strong absorption by acetone in this optically thick droplet is responsible for the saturation of the volume integrated signal, meaning a loss in sensitivity for the measurements (Fig. 16).

\subsection{Two-color Laser-induced fluorescence (2cLIF) thermometry}

Some organic dyes used in dye lasers like rhodamine B or sulforhodamine B combine relatively high fluorescence yields with strong temperature dependences of their fluorescent emissions (Coppeta and Rogers 1998) and therefore have been widely used for measuring the temperature in many kinds of liquid flows (Kim et al. 2003; Lemoine et al. 1999; Sakakibara and Adrian 1999, 2004). A small amount of these dyes, typically a few $\mu \mathrm{g} / \mathrm{L}$, is sufficient to generate a signal which can be used for measurement. In contrast to the fluorescent dopants used in laser-induced exciplex fluorescence thermometry, the dye molecules are usually only very slightly affected by quenching by oxygen and some of them resist relatively well to photobleaching. For further discussion, the signal intensity in Eq. (10) can be

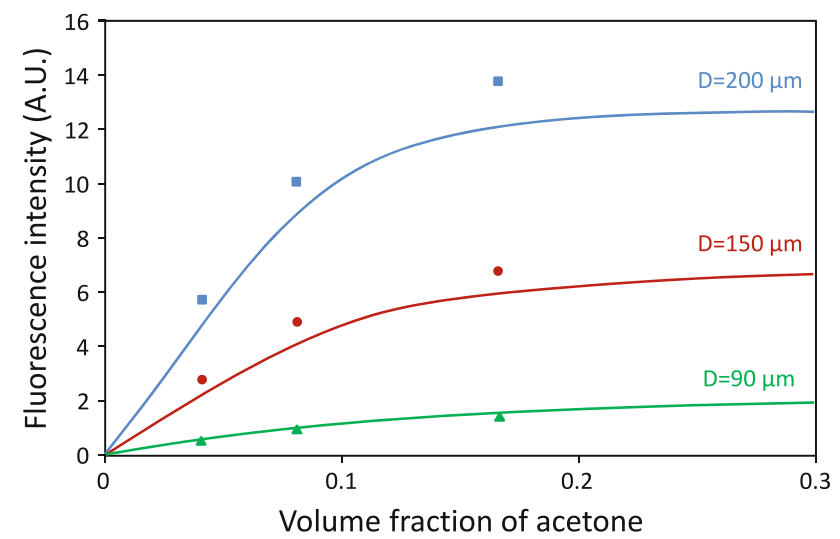

Fig. 16 Evolution of the intensity of the fluorescence signal of acetone with the volume fraction of acetone $Z_{\mathrm{a}}$ in the droplet. Comparison with the model based on ray tracing. Reprinted from Maqua et al. (2007)

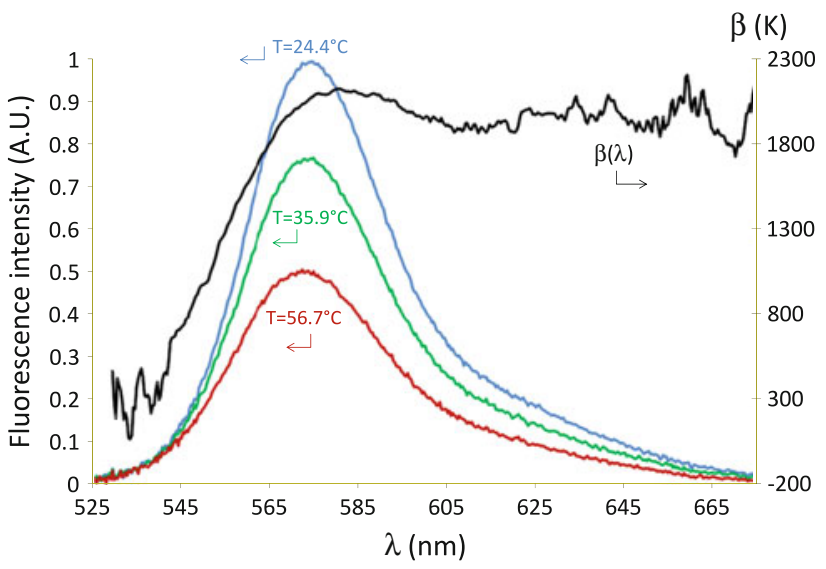

Fig. 17 Fluorescence spectra of rhodamine B dissolved in ethanol at different temperature. Wavelength dependence of the coefficient $\beta$ defined in Eq. (11). Reprinted from Lavieille et al. (2001)

rewritten in a form where the temperature $T$ appears explicitly (Lavieille et al. 2001):

$I_{f}(\lambda)=K_{\text {opt }}(\lambda) K_{\text {spec }}(\lambda) I_{0} C V_{c} \exp (\beta(\lambda) / T)$,

and where $\beta(\lambda)$ represents the spectral sensitivity to temperature. Parameters $K_{\mathrm{opt}}(\lambda), K_{\mathrm{spec}}(\lambda)$ and $\beta(\lambda)$ are not affected by a variation of the temperature. For some temperature-sensitive dyes (rhodamine B, sulforhodamine B, Pyromethene 597-8C9 or fluorescein FL27, etc.), a marked difference in the values of $\beta(\lambda)$ may exist between different spectral regions. This is illustrated in Fig. 17 for rhodamine $\mathrm{B}$ dissolved in ethanol. In this figure, values of $\beta$ are significantly lower in the yellow-green region of the fluorescence spectrum than in the red edge spectral region.

Based on experimental measurements, Lavieille et al. (2004) suggested the following expression for the fluorescence intensity $I_{f, i}$ detected over a specific spectral band noted $i$ : 


$$
\begin{aligned}
I_{f, i} & \approx K_{\mathrm{opt}, i} K_{\text {spec }, i} I_{0} C V_{c} f_{i}(T), \text { with } f_{i}(T) \\
& =\exp \left(A_{i} / T^{2}+B_{i} / T\right) .
\end{aligned}
$$

$A_{i}$ and $B_{i}$ are specific to a given combination of dye, solvent, excitation and detection wavelengths.

Lavieille et al. (2000) succeeded in using a single band of detection to measure the temperature of ethanol droplets flowing in air at room temperature. Measurements were made possible by the restrictive conditions in which the experiments were conducted. The droplet stream consisted of a line of monosized and periodically spaced droplets. The position of the droplets inside the probe volume could be adjusted in a very reproducible manner and maintained identical for all measurements. This means that any variations in signal intensity between different measurements could be attributed exclusively to the effect of temperature, i.e., $f(T)$ in Eq. (12). As already mentioned, the product $I_{0}$ $C V_{c}$ in Eq. (12) may be affected by various factors and changes when the droplet crosses the measurement volume formed by the intersection between the laser beam and the detection solid angle. The most common methods of eliminating the problem are based on intensity ratios in which one or two different dyes are utilized but a few alternatives to these ratiometric approaches do exist. For example, Hopkins et al. (2006) exploited the fact that the fluorescence spectrum of rhodamine B increases in width with the temperature when normalized by its maximum. Temperature is derived from the measurement of the width of the fluorescence spectrum at a quarter of its maximum intensity. This method based on the shape of the fluorescence spectrum was used to characterize the temperature of a train of alcohol/water droplets evaporating at a low ambient pressure.

\subsubsection{Two-color two-dye laser-induced fluorescence methods}

This method was pioneered in single-phase liquid flows by Coppeta and Rogers (1998) and substancially improved by Sakakibara and Adrian (1999). Two fluorescent dyes A and B with emission spectra which differed enough to permit the separation of their emissions by optical means were mixed with the liquid solvent. In the ideal case where the optical separation is perfect (i.e. no fraction of the light emitted by $\mathrm{A}$ is coming into the detection band for the light emitted by $\mathrm{B}$ and reciprocally), the ratio $R$ of the fluorescence intensities detected from each dye can be written as followed:

$$
\begin{aligned}
R_{A B}(T) & =\frac{I_{f, A}}{I_{f, B}}=K \frac{C_{A}}{C_{B}} f_{A B}(T), \text { with } K \\
& =\frac{K_{\mathrm{opt}, \mathrm{A}} K_{\mathrm{spec}, \mathrm{A}}}{K_{\mathrm{opt}, \mathrm{B}} K_{\mathrm{spec}, \mathrm{B}}} \text { and } f_{A B}=f_{A} / f_{B}
\end{aligned}
$$

To accurately measure temperature, Eq. (13) shows that the dyes need to have fluorescence emissions with very different temperature sensitivities, while the ratio of their concentrations $C_{A} / C_{B}$ should be kept constant and uniform in the flow. This latter requirement is not difficult to fulfill in droplet flows since organic dyes are heavy molecules which remain in liquids during the droplet evaporation. Coppeta and Rogers (1998) were the first to apply this approach for temperature and also for $\mathrm{pH}$ measurements. They pointed out the effects of spectral conflicts which arise from an overlap between absorption and emission bands. Sakakibara and Adrian $(1999,2004)$ carried out measurements with a similar approach in the field of thermal convection and studied the case where the separation of the fluorescence spectra is not perfect. Other examples can be found in literature in this field (Kim et al. 2003; Shafii et al. 2009; Sutton et al. 2008). To the best of our knowledge, the two-color two-dye approach was never considered for measuring the temperature of droplets even if there is no real restriction for that.

\subsubsection{Two-color single-dye laser-induced fluorescence methods}

As mentioned above, for many organic dyes, the fluorescence spectrum has been observed to not be uniformly affected by temperature (Fig. 17). This phenomenon remains currently mostly unexplained but does have some similarities with the blue shift and spectral deformations observed by Bai and Melton (1997). As suggested by these authors, one explanation could be that higher vibrational levels of the excited electronic state are populated when the temperature increases. The blue edge region of the fluorescence spectrum would then be enhanced because of the higher energy gap between the excited state and the ground state. According to Eq. (12), the ratio $R_{12}(T)$ of the intensities of spectral bands 1 and 2 is given by:

$$
\begin{aligned}
R_{12}(T) & =\frac{I_{f, 1}}{I_{f, 2}}=\frac{K_{\mathrm{opt}, 1} K_{\mathrm{spec}, 1}}{K_{\mathrm{opt}, 2} K_{\mathrm{spec}, 2}} f_{12}(T) \text { with } \\
f_{12}(T) & =f_{1}(T) / f_{2}(T)=\exp \left(A / T^{2}+B / T\right), A \\
& =A_{1}-A_{2}, B=B_{1}-B_{2}
\end{aligned}
$$

In this expression, $K_{\mathrm{opt}, i}$ and $K_{\mathrm{spec}, \mathrm{i}}$ depend on the arrangement of the detection system and the nature of the solvent. To accurately measure the temperature, spectral bands need to be selected so that the differences between coefficients $A_{1}$ and $A_{2}, B_{1}$ and $B_{2}$ are as great as possible. A reference measurement at a known temperature $T_{0}$ is necessary to eliminate the influence of system constants. Denoting $R_{0}$ the fluorescence ratio obtained in the reference measurement, Eq. (14) can be rewritten: 
$\ln \left(\frac{R}{R_{0}}\right)=A\left(\frac{1}{T^{2}}-\frac{1}{T_{0}^{2}}\right)+B\left(\frac{1}{T}-\frac{1}{T_{0}}\right)$.

In this equation, coefficients $A$ and $B$ are first determined by means of a calibration performed in a temperaturecontrolled test cell for example. The liquid temperature is then obtained by solving Eq. (15).

The first demonstration of the ratiometric method described by Eq. (15) was carried out by Lavieille et al. (2001) who studied the evaporation and the combustion of ethanol droplets. Since this pioneering work, the technique has been improved considerably for application in many different situations. Figure 18 shows a typical optical system used for point wise measurements. The excitation volume of the fluorescence can be created by one laser beam or by two crossing laser beams like in the 1-D LDV optical device featured in this diagram. This configuration offers the possibility of measuring the droplet temperature and velocity simultaneously. A fiber-coupled achromatic doublet allows the fluorescence emission to be collected preferentially at right angle. The optical signal traverses a holographic filter that blocks the laser light scattered by the droplets. The remaining fluorescence is then divided into two parts by a beamsplitter and optical filters which enable the fluorescence signal in the two spectral bands of interest to be separated. Finally, two photomultiplier tubes equipped with rapid pre-amplifiers detect the fluorescence photons. Another alternative to the forward scatter detection system shown in Fig. 18 is to use a single-probe backscatter configuration in combination with LDV measurements (Lavieille et al. 2000). Based on the optical setup in Fig. 18, signal processing can be performed in different ways depending on the resolution aimed at achieving.

Spatially averaged droplet temperature measurements: If global information is of interest, laser beams larger than the droplet size can be used to illuminate approximately the whole droplet volume. The signal intensity of each spectral band can be also averaged over the time corresponding to the transit of several droplets in the probe volume. As explained above, the average temperature resulting from this process may differ significantly from the arithmetic average mainly because of the non-uniform distribution of the fluorescence excitation field within the droplets. In general, the differences with the arithmetic volume average are greater with stronger thermal gradients and are located near the droplet surface. However, since the temperature distribution is unknown a priori, these differences remain difficult to quantify in practice. To obtain a comparison with experiments, averaging by optics can be applied to theoretical temperature fields obtained by modeling the droplet heating and evaporation (Maqua et al. 2006). Spatially averaged measurements were mainly applied to study lines of monosized and periodically spaced droplets. The droplet temperature was characterized for many liquids in situations including droplet heating in flames (Castanet et al. 2005b, c; Lavieille et al. 2001), droplet heating in a hot air stream (Castanet et al. 2007; Deprédurand et al. 2010), droplet heating in the thermal boundary layer of a hot wall (Castanet et al. 2002), droplet heating when colliding with a hot wall (Castanet et al. 2009) and droplet cooling in ambient air (Lavieille et al. 2000, 2002). The same method was used to measure the liquid temperature in a spray of decane droplets convected in an overheated turbulent gas flow (Depredurand et al. 2011) and in a high-pressure diesel spray (Wolff et al. 2007). In the latter study, the signal acquisition was triggered on the injection cycle to enable phase-locked monitoring of the liquid phase temperature. Temperature maps of the spray resulting from these measurements reveal how the droplet temperature is related to the injection pressure due to the friction forces inside the injector.

Imaging systems: An extension to imaging of the singledye two-color LIF thermometry was covered in several
Fig. 18 Two-color laserinduced fluorescence optical system. Reprinted from Depredurand et al. (2011)

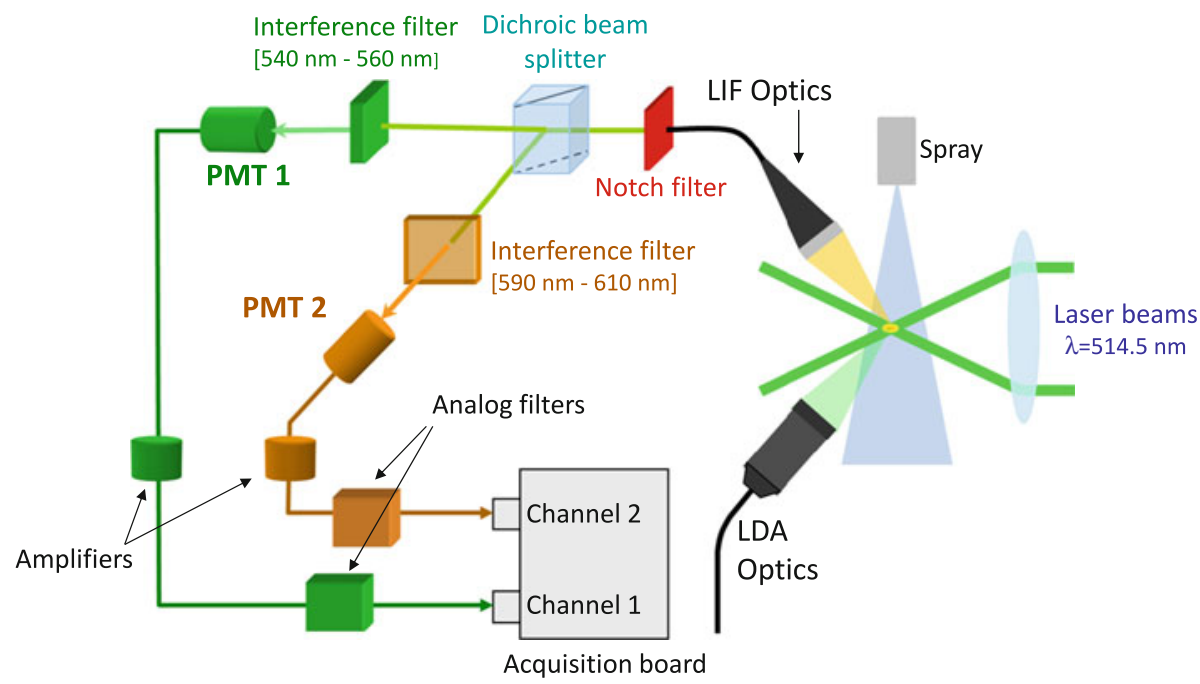


publications. Bruchhausen et al. (2004) were the first to adapt the single-dye ratiometric approach using two cameras mounted behind a beam splitter to obtain two images of the same view at different wavelengths, in the case of an overheated turbulent jet. However, these measurements did not concern droplets but heat convection in a turbulent liquid jet. Previously, similar optical arrangements had also been used in relation with the already described two-dyes two-color LIF approach (Kim et al. 2003; Sakakibara and Adrian 1999). Regarding droplets and sprays, Duwel et al. (2007) reported temperature measurements of burning ethanol droplets seeded by rhodamine B. In this work, two intensified CCD cameras were positioned on both sides of a spray burner. Because of the camera's large field of view, pixel-by-pixel correspondence between the two cameras does not seem to have been a critical issue in this study. Recently, Vetrano et al. (2012) presented an application of 2 cPLIF thermometry to characterize the temperature variations of ethanol droplets produced by flash-boiling atomization. PLIF was also used by Dunand et al. (2012, 2013) to characterize the temperature of droplets impinging onto a wall heated above the Leidenfrost temperature. As 2cLIF techniques are insensitive to the shape of the liquid volume in principle, they are particularly well adapted to handle this complex situation where droplets can be strongly deformed. In the work of Dunand et al. (2012), temperature measurements within streams of monosized droplets were achieved at a very small spatial scale using a long distance microscope. One difficulty pointed out by the authors was the sensitivity to the pixel-to-pixel correspondence of the images of the two cameras. Image correspondence needs to be optimized at a sub-pixel level before calculating the intensity ratio to avoid artifacts. Figure 19 shows an example of temperature measurement achieved in the case of splashing. As we can see in Fig. 19a, water drops coming from the right broke up into very fine droplets and a significant increase in the liquid's temperature was observed at the drop impact (Fig. 19c).

Local temperature measurements within droplets: The measurement system presented in Fig. 18 can be also modified in order to obtain the temperature distribution inside the droplet (Castanet et al. 2003b, 2005a, 2011a, b). This is only possible in the case of monodisperse droplet streams as a result of the large number of droplets that can be sampled and their high reproducibility. In this setup, optics needs to be selected in order to create a probe volume much smaller than the droplet volume, while the sampling period of the fluorescence signal is fixed at a very small value compared to the transit time of the droplets through the measurement volume. When a droplet crosses the laser beams, different zones of the droplet interior are successively illuminated. The intensity ratio and thus the temperature are calculated at each location. The injector is shifted several times by a few microns and the droplets are scanned again at a further position. The superimposition of the scan profiles allows reconstructing the internal temperature provided a perfect periodicity of droplets in the stream. This reconstruction should account for the lens effect at the droplet interface. The internal excitation field is calculated using either geometrical optics or Generalized Lorentz-Mie Theory (GLMT). An example of temperature maps is presented in Fig. 20. In this example, the lens effect at the droplet interface was corrected using geometrical optics. The outskirt of the droplet remains mostly blank which reflects the lower contribution of this region to the signal due to the lens effect. Similar results were also obtained with the use of GLMT (Castanet et al. 2005a). The method described above was used to investigate the heating of droplets of about $200 \mu \mathrm{m}$ in diameter, i.e., about 10 times the dimensions of the measurement volume. Internal convection was identified as a key element which explains the temperature distribution obtained in these (a)

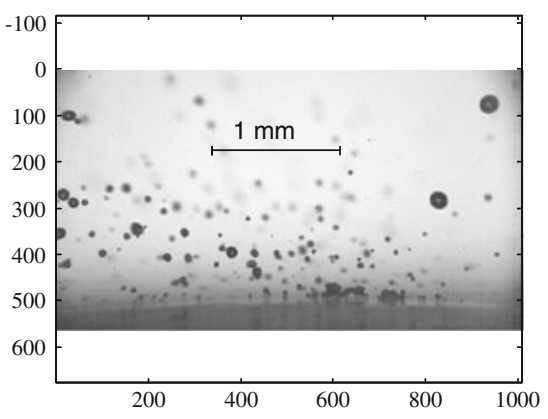

(b)

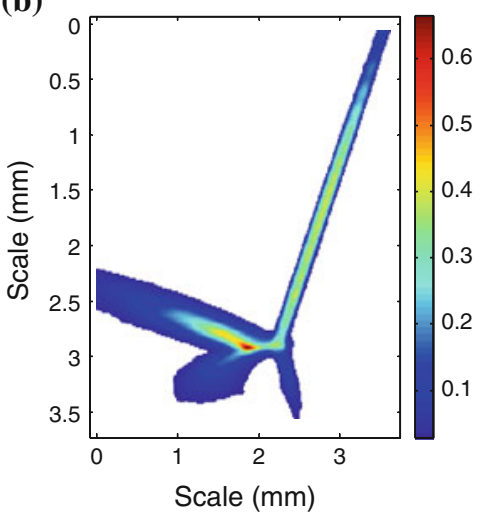

(c)

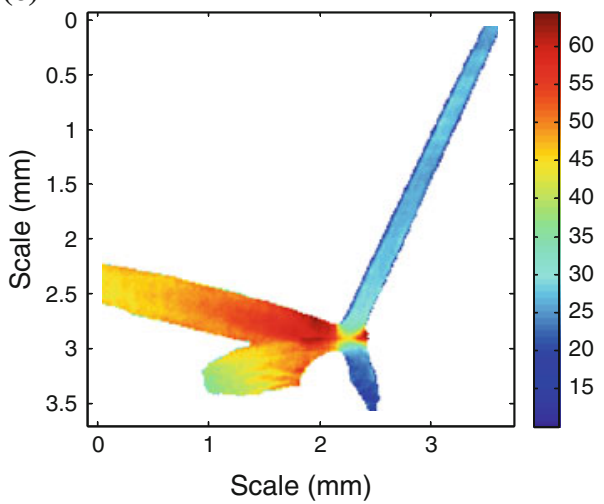

Fig. 19 An example of 2cPLIF temperature measurement in the case of a splashing (a image of the droplets taken by shadowgraphy, $\mathbf{b}$ timeaveraged fluorescence intensity field in one band, $\mathbf{c}$ temperature distribution). Reprinted from Dunand et al. (2013) 
Fig. 20 Comparison between theoretical and experimental temperature maps (case of an n-decane droplet $D=190 \mu \mathrm{m}$, $V=6.7 \mathrm{~m} / \mathrm{s}$, inter-droplet distance $=2.7 \mathrm{D}$, temperatures are in ${ }^{\circ} \mathrm{C}$, time in $\mathrm{ms}$, horizontal and vertical scales are in microns). Reprinted from Castanet et al. (2011b)
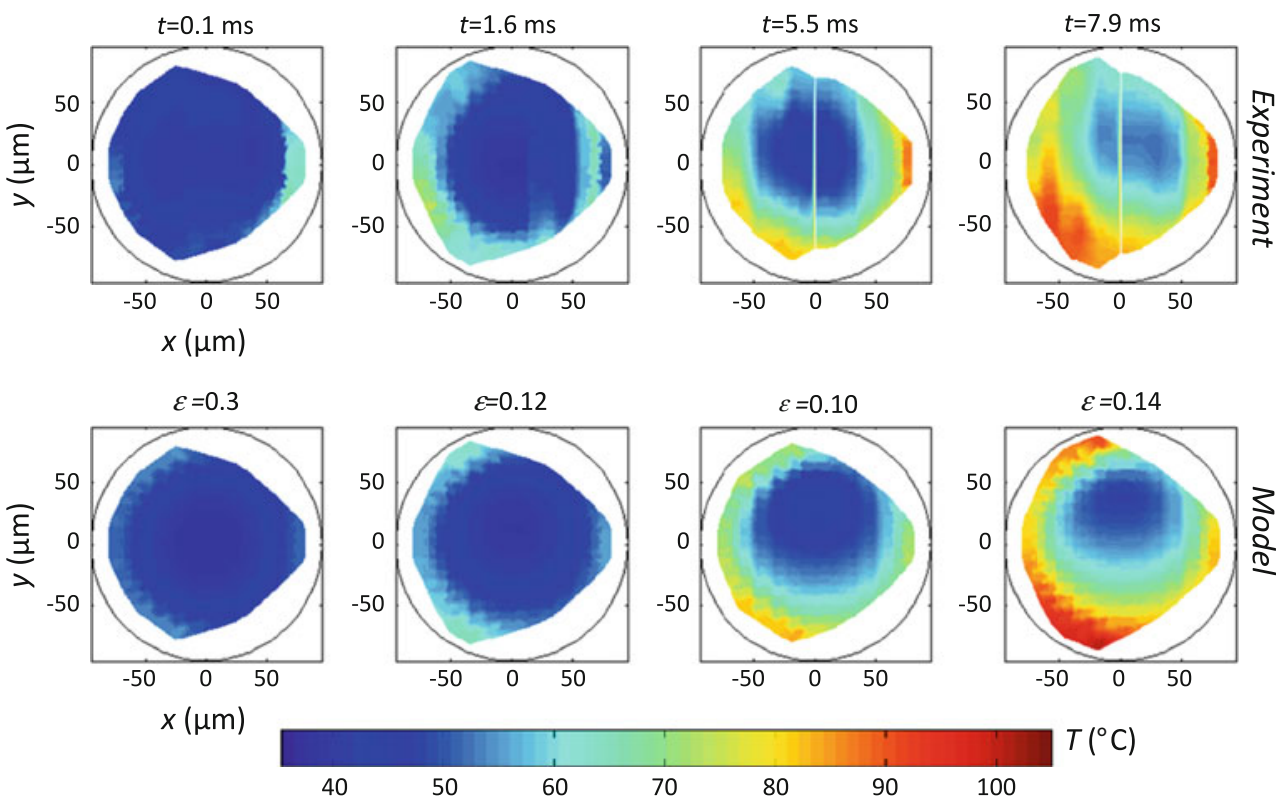

measurements. An internal circulation pattern similar to a Hill's spherical vortex is assumed to be responsible for a stronger heating of the trailing edge than the leading edge. Comparisons between measurements and numerical simulations have shown how the magnitude of the velocity in the internal flow depends on gas friction, the distance between the droplets and the Marangoni effect (Castanet et al. 2011a, b).

Temperature measurements of multicomponent droplets: Probing the temperature of multicomponent droplets with 2cLIF thermometry requires special care, and the selection of an appropriate fluorescent dye is of great importance. The possibility with different liquids without changing the spectral bands of detection should be also considered. The chemical environment, i.e., mainly the solvent composition, alters the spectroscopic properties of the dye and particularly modifies collisional quenching energy redistribution in the excited state and therefore also some parameters involved in the expression of the fluorescence signal such as $K_{\text {spec }}$ or temperature sensitivity coefficients $A$ and $B$ in Eq. (12). For example, the fluorescence of rhodamine B or sulforhodamine B varies significantly according to the solvent composition which limits their usefulness for the study of multicomponent droplets. The composition of multicomponent droplets may change considerably during an experiment due to the differential vaporization of the liquid components. Maqua et al. (2006) used rhodamine B to measure the temperature of ethanol/ acetone droplets. To deal with composition changes, they added a third band of detection to the optical setup presented in Fig. 18. This third band enabled the calculation of a second fluorescence intensity ratio whose sensitivity to temperature and composition differed from the first ratio.
Although the method can theoretically be used to determine both temperature and liquid composition, it proved to be poorly sensitive to composition and to require considerable effort for it to be calibrated for both temperature and composition. Furthermore, it seems difficult to adopt the same method to deal with mixtures containing more than two species. More recently, Depredurand et al. (2008) demonstrated that the emission of pyrromethene 597-C8 is almost unchanged when dissolved in many different liquids such as alcohols, ketones and alkanes and their mixtures. These authors exploited this behavior to measure the temperature of several mixtures including ethanol/3pentanone, decane/3-pentanone (Depredurand et al. 2008, Sazhin et al. 2011). Although pyrromethene 597-C8 is weakly soluble in aqueous solutions, its use may allow to generalize the two-color LIF approach to complex mixtures containing a large set of chemical species.

Temperature measurements in sprays: Several studies have more specifically addressed the implementation of the 2cLIF technique in sprays (Bruchhausen et al. 2006; Depredurand et al. 2011; Labergue et al. 2010, 2012a, b; Wolff et al. 2007). These studies describe how the 2cLIF technique, initially used and validated in the case of single calibrated droplets, should be modified for reliable measurements in sprays. Although the droplet size is normally canceled out in the intensity ratio (Eq. 14), a residual influence of the droplet size on the intensity ratio was pointed out by Labergue et al. (2010). In relation with this effect, they also noticed that the fluorescence spectrum was modified in the blue edge region (Fig. 21). The deviation from the fluorescence spectrum emitted by the same molecules dispersed in a cell is all the more important than the droplets are small. The origin of this effect is still unclear 


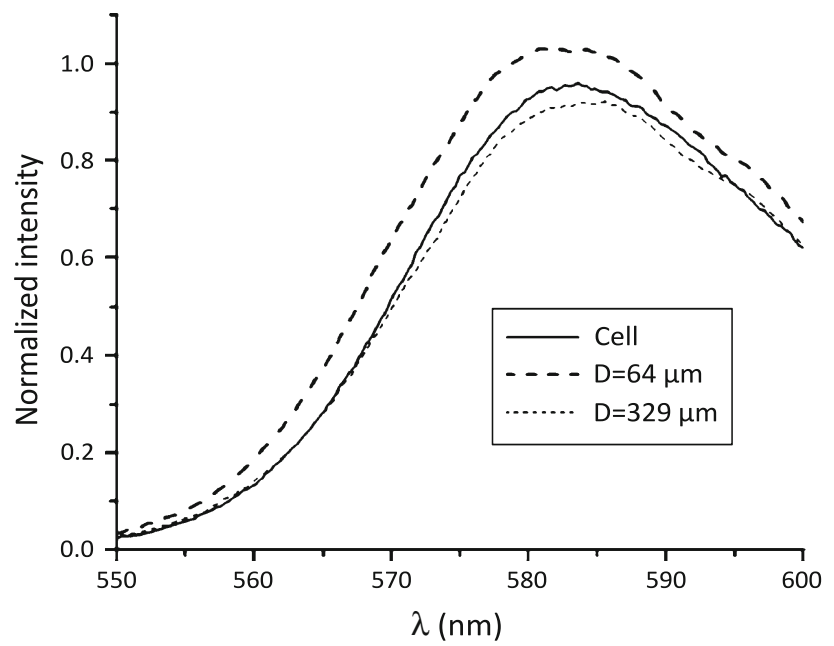

Fig. 21 Fluorescence spectra recorded in a cell and in droplets of calibrated sizes. Reprinted from Labergue et al. (2010)

even if potential explanations were provided in Labergue et al. (2010). Similar size-dependent variations of the intensity ratio were also reported with different dyes dissolved in different liquid solvents (Depredurand et al. 2011; Dunand et al. 2012). Depredurand et al. (2011) showed that the size effect is drastically reduced when the dye concentration is increased. A high concentration in dye was also used by Wolff et al. (2007) to perform measurements in high-pressure diesel sprays. However, in this work, high concentrations in dye were initially a mean of suppressing MDRs clearly visible both in the red and blue edges of the emission spectrum from droplets seeded with pyrromethene 597-C8. An intrinsic correction for the size effect, i.e., one that does not require prior knowledge of the droplet size, was proposed by Labergue et al. (2010). It consists of the addition of a third spectral band of detection which is particularly sensitive to the spectral influence of the droplet size.

In dense sprays, scattering of the laser light by the droplets may be strong enough that droplets situated far from the focal plan of the detection system can be illuminated and can contribute to the global fluorescence signal. This was identified as a potential source of error in addition to the afore-mentioned spectral effect of the droplet size (Labergue et al. 2012a). The problem can be partially overcome by using detection optics with a very small depth of field like long distance microscopes (Labergue et al. 2012a, b). Recently, measurements of droplet temperature per class of droplet size were reported by Depredurand et al. (2011). This was made possible by combining two or three-color LIF and phase Doppler analysis (PDA). For that purpose, the acquisitions of PDA and LIF signal were synchronized on the same time base. The combined PDA/LIF technique was demonstrated by Depredurand et al. (2011) on a spray of n-decane injected

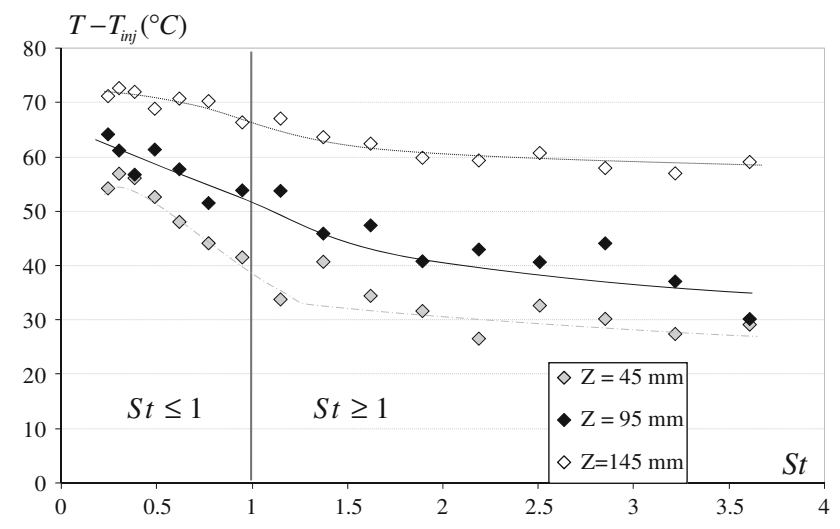

Fig. 22 Evolution of the droplet temperature as a function of the stokes number for three downstream locations on the flow centerline ( $Z=45 \mathrm{~mm}, Z=95 \mathrm{~mm}$ and $Z=145 \mathrm{~mm}$ ). Reprinted from Depredurand et al. (2011)

at ambient temperature in an overheated turbulent air flow. Joint measurements of the droplet size, velocity and temperature allowed the researchers to quantify the effect of the stokes number based on the integral turbulence length scale on the droplets heating. As illustrated in Fig. 22, as the smallest droplets have low stokes numbers and are transported by the turbulent structures, they have higher heating rates than bigger droplets. Labergue et al. (2012b) also used combined 3cLIF/PDA measurements to study the mixing of two sprays injected at different temperatures.

\subsection{Techniques based on the fluorescence lifetime}

The molecular fluorescence lifetime is an intrinsic characteristic of fluorescent species which is only affected by their chemical environment. Its measurement can be used to obtain environmental information with fewer precautions than in the case of time-integrated fluorescence intensities measurements which are dependent on experimental factors such as excitation intensity and optical collection efficiency. Recently, the potential of this kind of approach has been demonstrated in micro-fluidics (Casadevall i Solvas et al. 2010, 2011; Zeng et al. 2011). These studies concern the mixing of reagents in droplets immersed in a second immiscible liquid but could certainly be transposed to other situations including droplets flowing into an external gas. Monosized water-in-oil droplets are generated at frequencies of about $1 \mathrm{kHz}$ from two aqueous solutions flowing in parallel into a T-junction against an oil phase flow (Fig. 23). To make use of the fluorescence, two different fluorescent dyes are introduced in the solutions. Their lifetime should be different enough to separate their signals in the time domain. The fluorescence decay of the mixture takes on a biexponential form. In order to extract fluorescence lifetime, a timecorrelated single-photon counting (TCSPC) methodology 

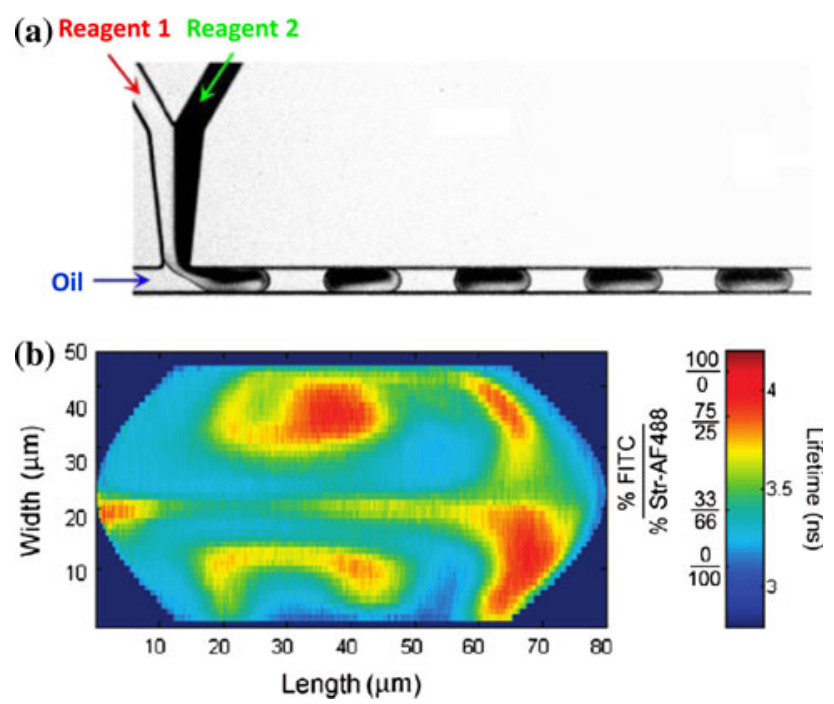

Fig. 23 Lifetimes measurements in monosized water-in-oil droplets. a On chip encapsulation in droplets of two originally separate aqueous reagents that fluoresce (FITC and Str-AF488). Reprinted from Casadevall i Solvas et al. (2011). b Two-dimensional maps of mixture fraction and average lifetimes within droplets with $1 \mu$ s time resolution (a ratio of $33 \%$ FITC/66 \% Str-AF488 represents a perfectly mixed solution). Reprinted from Casadevall i Solvas et al. (2010)

combined with confocal microscopy is carried out to record fluorescence data in the time domain. Femtosecond or picosecond pulse lasers operating at several $\mathrm{MHz}$ are used to provide single or two-photon excitation. The objective lens of the confocal microscope brings the laser light to a tight focus to probe volumes down to a few picoliters. The droplets are scanned line-by-line by moving the focal point a few microns across the channel. The periodically generated droplets are identical, which means cross-sectional images of the droplets can be built by aligning the scanning lines across the channel. Figure 23 shows an example of average lifetime map which clearly reveals the mixing generated by circulations within droplets.

Post-processing of the data may be complex, especially statistical analysis. Data first need to be deconvoluted with the instrument response function of the detector (IRF). Casadevall i Solvas et al. (2010) used a maximum likelihood estimator to determine the average lifetime of the mixture also related to the fraction of each component. To maintain the data within the limits of an acceptable error when aiming to reduce the time resolution, they found it necessary to increase the number of analyzed droplets in proportion to maintain the same cumulative count in all channels. By adding up photons from thousands of droplets (which requires several minutes), they demonstrated that images of fluorescence lifetime within micro-droplets could be constructed with a time resolution of $1 \mu \mathrm{s}$ which in fact reveals the spatial resolution. In the work of Zeng et al. (2011), a purposed made method was developed to accurately calculate the mixing ratios.
Obviously, other information than composition can be extracted from lifetime measurements. Significant thermal dependences of the lifetime was reported for many substances, including organic dyes and thermographic phosphors (Allison and Gillies 1997). Some studies also demonstrated that the fluorescence lifetime can be used to measure $\mathrm{pH}$ and viscosity (Blatt et al. 1981; Lakowicz and Szmacinski 1993). Other techniques than the afore-mentioned TCSPC can be used to determine the fluorescence lifetime working with either the time domain or the frequency domain. Time-correlated single-photon counting (TCSPC) is usually used because variations in source intensity and photoelectron amplitudes can be ignored. Furthermore, the time resolution can be reduced down to a few ps. Also in the time domain, gating methods can be considered but with a more limited temporal resolution. Implementation can involve gated integrators and boxcar averagers or a delay generator controlling a gated optical intensifier (GOI) placed in front of a CCD detector which acts both as a very fast shutter and an intensifier to amplify the light signal. A few examples of such applications of time-gated CCD cameras are mentioned in the section concerning thermographic phosphors. In the frequency domain, the sample is excited by a modulated source of light. The fluorescence emitted by the sample has a similar waveform but is modulated and phase-shifted from the excitation curve.

\subsection{Laser-induced exciplex fluorescence (LIEF)}

Laser-induced exciplex fluorescence (LIEF) is a particular case of LIF. The photophysics behind LIEF and its applicability for probing droplet temperature are summarized in the following. One of the additives, called the monomer $M$, absorbs light from a laser source to form the first excited singlet state $M^{*}$ :

$M+h v \rightarrow M^{*}$

The interaction of $M^{*}$ and an appropriately chosen ground state molecule $G$ (initially added with $M$ in the liquid) leads to the formation of the exciplex $E^{*}$ (excited state complex also called an excimer in the special case where $G=M$ ) following the reversible reaction:

$M^{*}+G \leftrightarrow E^{*}$

The exciplex formation can only take place if the monomer in its excited state $M^{*}$ finds a reaction partner $G$ within the short lifetime of its excited state (typically only a few nanoseconds). No binding is possible if the monomer is in the ground state. Given that the frequency of molecular collisions is higher in a liquid than in a gas, the 
probability of forming an exciplex is only significant in the liquid. Another key feature for practical applications relates to the fact that the fluorescence emission of exciplex complexes is red-shifted compared to the monomer due to the binding energy in the exciplex with respect to separated $M^{*}$ and $G$. This behavior is illustrated in Fig. 24 for the system naphthalene (N)/tetramethyl-pphenylenediamine (TMPD), in which the monomer TMPD* has an emission centered around $400 \mathrm{~nm}$ and the exciplex $E^{*}$ at about $500 \mathrm{~nm}$ (Murray and Melton 1985).

As a result of the red-shifted exciplex emission, the fluorescence emissions of the monomer and the exciplex can easily be separated with adapted bandpass filters. In many applications of LIEF to droplet measurements, this feature is used to discriminate between the liquid and gaseous phases. Ultimately this has been exploited for quantifying the amount of liquid still present in evaporating sprays (Desantes et al. 2005; Fansler et al. 2009; Felton et al. 1993; Fujimoto et al. 2002; Ghandhi et al. 1994). In works dealing with vapor/liquid visualization, an excess of the ground state partner $G$ is usually introduced to suppress the monomer in the liquid in favor of the exciplex (Melton 1983). The fluorescence emitted by the droplets is thus predominantly from the exciplex $E^{*}$, while the fluorescence from the vapor phase is almost exclusively from the monomer $M^{*}$. Particular issues for the optical separation are the temperature dependence of the formation of the exciplex and the possibility of remaining monomers in the liquid. While these problems need to be suppressed for quantitative liquid/vapor visualization, they are actually an advantage for probing the temperature in the droplets. The derived technique called exciplex fluorescence thermometry (EFT) was first demonstrated by Murray and Melton (1985).

The chemical equilibrium described by Eq. (17) is sensitive to the temperature with its equilibrium constant being about proportional to $\exp (-\Delta G / R T)$, while $\Delta G$ is the binding free energy with respect to separated $M^{*}$ and $G$. Increasing the temperature shifts the equilibrium toward the monomer $M^{*}$.On this basis, the ratio of the exciplex emission intensity $I_{E}$ and the monomer emission intensity $I_{M}$ (equivalent to the ratio of the concentrations $\left[\mathrm{E}^{*}\right] /\left[\mathrm{M}^{*}\right]$ ) are expected to decrease with temperature. This can be easily verified in Fig. 24 for the $1.0 \% \mathrm{~N} / 2.5 \% \mathrm{TMPD} /$ Hexadecane system between 140 and $265^{\circ} \mathrm{C}$ (but the change is quite modest between 25 and $140{ }^{\circ} \mathrm{C}$ ). In the EFT technique, the liquid temperature is derived from the measurement of the intensity ratio $I_{E} / I_{M}$. As this technique is based on relative intensities like the $2 \mathrm{cLIF}$ thermometry, it is not affected by experimental factors such as laser intensity or the droplet position in the probe volume. Like 2 cLIF thermometry, a calibration is required to infer the temperature from the intensity ratio although calibration curves can have a more complex appearance than one would expect from the previous description. The evolution of the intensity ratio with the temperature may not be monotonic in the temperature range of interest as illustrated in Fig. 25 for the $1.0 \% \mathrm{~N} / 2.5 \% \mathrm{TMPD} / \mathrm{n}$-decane system.

In fact, the binding reaction described in Eq. (17) can only be treated as a chemical equilibrium at sufficiently high temperatures. At low temperatures, the exciplex's rate of formation may not be fast enough for the equilibrium to be obtained within the lifetime of $M^{*}$ resulting in a more reduced exciplex production and emission. The nonmonotonous shape of the calibration curve has several drawbacks. The technique suffers from a loss of sensitivity in the temperature region where the sign of the slope of the calibration curve changes. It also creates an ambiguity in converting the intensity ratio into temperature. The errors resulting from these adverse effects were quantified by Zhang and Melton (1993) for the experiments conducted by Hanlon and Melton (1992).

The dependence of the intensity ratio on the concentration of the dopant is a particular issue for measurements.

Fig. 24 Influence of the temperature on the fluorescence emission of the $1.0 \% \mathrm{~N} /$ $2.5 \%$ TMPD/Hexadecane excited at $308 \mathrm{~nm}$. Reprinted from Murray and Melton (1985)

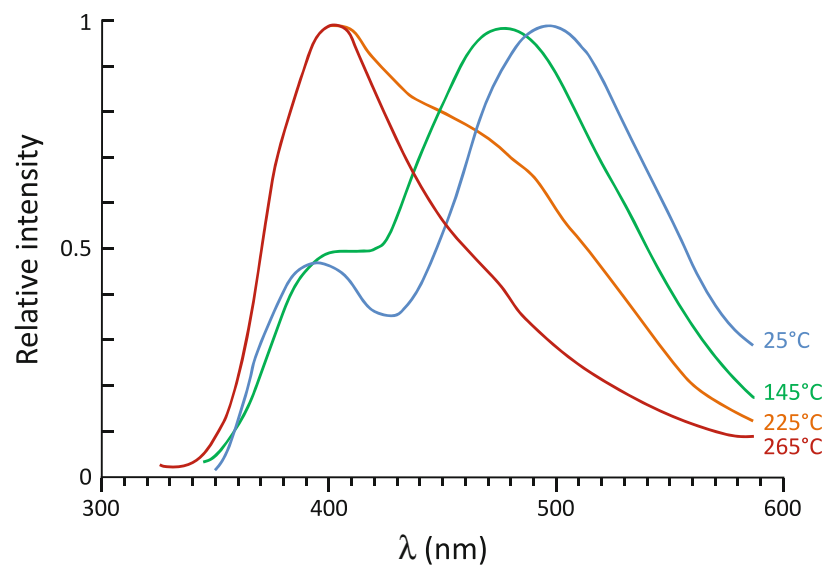




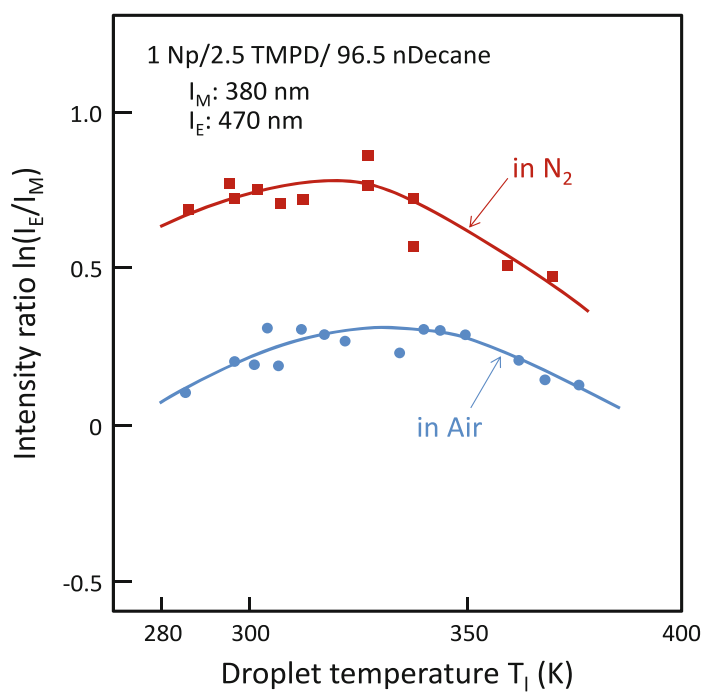

Fig. 25 Variation of the intensity ratio $I_{\mathrm{E}} / I_{\mathrm{M}}$ with the temperature for the $1.0 \% \mathrm{~N} / 2.5 \% \mathrm{TMPD} / \mathrm{n}$-decane system. Reprinted from Kadota et al. (1991)

Increasing the concentration in $\mathrm{G}$ will shift the equilibrium toward $\mathrm{E}^{*}$. However, in an evaporating droplet, the concentration of the additive may significantly change which is likely to modify the position of the chemical equilibrium in Eq. (17) and the calibration curve obtained at a fixed composition will no longer be appropriate. Wells and Melton (1990) only partially avoided the problem by using a particular case, wherein the ground state partner $G$ and the monomer $M$ were the same molecule namely pyrene. However, since pyrene evaporates at a lower rate than the decane in which it was dissolved, the increase in pyrene concentration was suspected to have resulted in erroneous measurements. To assess the validity of their measurements, Wells and Melton (1990) had to at least verify that the droplets were undergoing minimal changes in size at the highest ambient temperatures. Drop sizing was also used for verification by Escobar et al. (2001)in their temperature measurement of ethanol droplets using pyrene as dopant. In this study, changes in pyrene concentration could be ignored essentially because measurements were performed at very short distances downstream of the injection.

Concentration would not be an issue if tracers almost coevaporate with the liquid fuel. Wells and Melton (1990) used fluorobenzene/DEMA in n-hexane solution as it is one of the few examples of a mixture with a known co-evaporation of all the components.

Gossage and Melton (1987) succeeded in finding a concentration-independent thermometric dopant 1,3-bis $\left(1^{\prime}\right.$ pyrenyl) propane (PYPYP) seeded in hexadecane. PYPYP was used for probing the temperature of droplets heated in a high temperature chamber either dissolved in hexadecane
(Hanlon and Melton 1992) or in decane (Lu and Melton 2000). Also in the work of Hanlon and Melton (1992), the risk of concentration change due to evaporation was very limited because the droplets were made of hexadecane which has a high boiling temperature.

Additives usually accounts for several percent in the EFT method which can potentially affect the evaporation and the heating of the droplets. In the work of Wells and Melton (1990), the absorption by pyrene was so strong that the light excitation was limited to the outskirts of the droplets and thus the temperature determined was more like a surface temperature than a volume average temperature. The other interest of the above-mentioned intramolecular exiplex PYPYP was that the dopant can be used at much lower concentrations than those required generally for intermolecular systems (only a few ppm of the droplet volume). In return, optical thickness effects due to bulk absorption could be eliminated (Gossage and Melton 1987). This meant Lu and Melton (2000) could characterize the temperature field within a $500-\mu \mathrm{m}$-size decane droplet using an imaging method. Megahed (1993a, b) used the same dopant to obtain two-dimensional temperature maps of hexadecane sprays injected in the temperature and pressure conditions of a diesel engine.

It should be noted that the previously described applications were all performed in pure nitrogen to avoid the quenching by oxygen. It is recommended to bubble nitrogen through the liquid or to degas the liquid (Escobar et al. 2001; Hanlon and Melton 1992; Lu and Melton 2000). The effects of oxygen on the fluorescence of both exciplex and monomer have been investigated in many studies (Kadota et al. 1991; Megahed 1993a; Wells and Melton 1990) which all show that the intensity ratio $I_{E} / I_{M}$ is drastically reduced in presence of oxygen, implying that the exciplex exhibits greater quenching by the oxygen than the monomer (Fig. 25). Winter and Melton (1990) used the quenching of naphthalene by oxygen to study the internal circulation within decane droplets. They carried out imaging experiments in which a thin laser sheet was moved a certain distance from the droplet center. Regions rich in oxygen appear as dark zones in their images. Quantitative information about the oxygen distribution could be obtained by normalizing the images taken in presence of oxygen against images of identical droplets recorded in pure nitrogen (Fig. 26).

Because of strong quenching by oxygen, EFT has not been applied to combustion except in the study of Matsumoto et al. (1999) who characterized the temperature of individual fuel droplets burning under microgravity in a drop shaft. However, the reliability of their measurements can be questioned-the change in dopant concentration during the droplet evaporation caused by quenching by oxygen should have been more thoroughly investigated. 


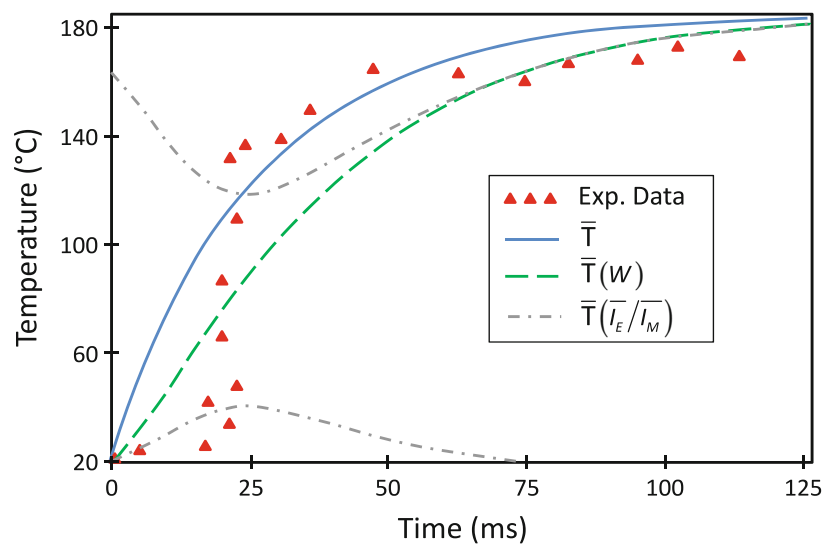

Fig. 26 Temporal evolution of the temperature of hexadecane droplet heating when falling into a hot tube. Measurement from Hanlon and Melton (1992) and comparison with simulation taken or not into account the error introduced by optical weighting (nonuniform excitation and absorption). Reprinted from Zhang and Melton (1993)

\section{Alternative measurement techniques}

\subsection{Morphology-dependent resonances}

Morphology-dependent resonances (MDRs) were used for measuring droplet sizes and evaporation rates as small as $10^{-4} \mathrm{~cm}^{2} / \mathrm{s}$ in continuous droplet streams (Chen et al. 1993; Swindal et al. 1996). In these measurements, the droplets were rather small, typically of around $25 \mu \mathrm{m}$. The use of MDRs to probe the temperature of single droplets is less common but has been reported in literature in this field (Chen et al. 1996; Mazumder et al. 1995a, b). The droplets were seeded by a thermochromic absorber and a lasing dye. The thermochromic absorber was $\mathrm{CoCl}_{2} \cdot \mathrm{H}_{2} \mathrm{O}$ dissolved in droplets made of isopropanol (85\%) and water $(15 \%)$. The thermochromism, corresponding to a change in the color with temperature, was found to be a consequence of a temperature-dependent configurational change of $\mathrm{Co}^{2+}$ complexes in the presence of $\mathrm{Cl}^{-}$ions. The absorption of the thermochromic additive is sensitive to temperature, i.e., when the temperature increases, the absorption around $670 \mathrm{~nm}$ also increases. The lasing dye added to the solution was rhodamine $\mathrm{B}\left(c=5 \cdot 10^{-4} \mathrm{~mol} / \mathrm{L}\right)$. A droplet acts as an optical micro-cavity for some specific fluorescence wavelengths located in the tail of the fluorescence spectrum and lasing can occur for designated wavelengths located in this spectral region. For lasing to occur in a droplet, the gain must compensate the total losses which include leakage loss, self-absorption by rhodamine $\mathrm{B}$ and absorption by the thermochromic additive. The leakage losses were very small for the specific wavelengths corresponding to MDRs in the droplet. The optimum lasing wavelength was about $635 \mathrm{~nm}$ for rhodamine $\mathrm{B}$ at $20^{\circ} \mathrm{C}$.

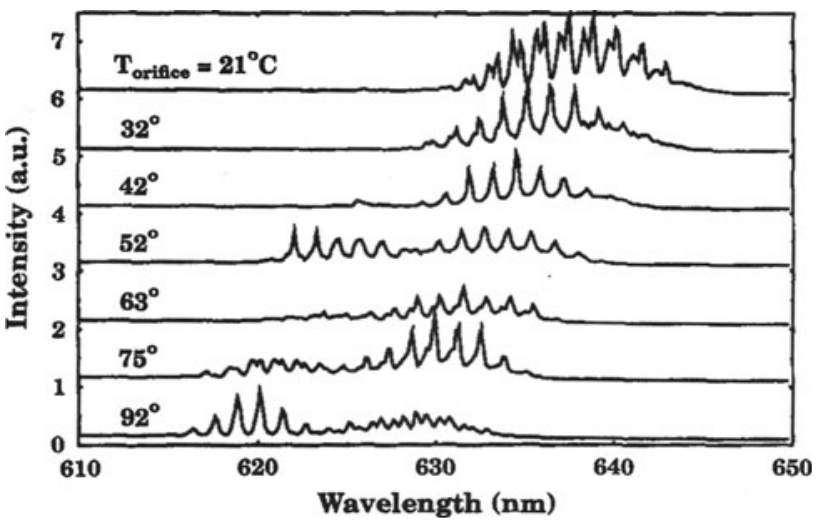

Fig. 27 Lasing spectra from droplets (diameter $70 \mu \mathrm{m}$ ) made of $85 \%$ isopropanol and $15 \%$ water seeded with $5.10^{-4} \mathrm{~mol} / \mathrm{L}$ rhodamine $\mathrm{B}$ and $\mathrm{CoCl}_{2} \cdot \mathrm{H}_{2} \mathrm{O}$ for different temperatures. Reprinted from Mazumder et al. (1995b)

A noticeable feature for the measurements was the blue shift of the lasing spectrum of the dye with the droplet temperature (Fig. 27). This phenomenon was due to increased absorption by the thermochromic absorbers when the droplet temperature increased. Absorption by the thermochromic additive must be compensated by a higher gain of the stimulated emission process. Consequently, the optimum lasing wavelengths were shifted to the blue. The droplet temperature can be inferred from the position of the lasing lines in the spectrum. In practical terms, droplets were illuminated by a pulsed Nd:YAG laser at $532 \mathrm{~nm}$. Spectra of rhodamine B were recorded at a $90^{\circ}$ angle by an imaging-spectrograph CCD system. An initial calibration of the position of the lasing lines in the spectrum as a function of temperature was necessary for a given droplet size.

\subsection{Thermographic phosphors}

Phosphors are solid materials which emit light when exposed to radiation. There are hundreds of different phosphors, each with its own luminescence properties (emission spectrum, characteristic emission time, etc.). Phosphors can be both organic and inorganic but those known to have an application in thermometry are mostly inorganic. Inorganic phosphors are made of a host material (such as a metallic oxide,...) doped with a small amount of an activator, mostly a rare earth compound. This activator forms luminescent centers where the excitation-emission process of the phosphor takes place. The thermal dependence certain phosphors' luminescence was exploited for many years, especially to remotely probe the surface temperature of wall, because they provide a non-contact, emissivity-independent, optical alternative to standard temperature sensors (Allison and Gillies 1997). Their ability to tolerate harsh conditions is another advantage. In 
combustion engines, appropriate inorganic phosphors can operate at temperatures up to $2,000 \mathrm{~K}$ and it is also commonly accepted that their luminescence properties are almost independent of pressure (Aldén et al. 2011). Thermographic phosphors are usually in the form of a fine powder (typically ranging from 1 to $10 \mu \mathrm{m}$ ). In measurements of wall surface temperature, a phosphor coating is applied to the surface of interest while in droplet applications, phosphor particles are simply dispersed into the liquid prior to its injection. In some cases, a surfactant is added to avoid the particles to agglomerate within the droplets or prior to the injection (Omrane et al. 2004c). As with LIF thermometry, two main approaches can be employed to infer the temperature from the light emission of thermographic phosphors: lifetime methods and two-line (or two-color) methods based on the intensity ratio of two or more emission bands. It should be noted that the emission spectrum of phosphors usually consists of several bands, sometimes with several discrete lines. In practice, lifetime measurements are mainly utilized with phosphors having time decays in the order of $\mu \mathrm{s}-\mathrm{ms}$. These relatively long lifetimes permit the use of conventional photomultiplier tubes and fast-gated cameras to capture the decay.

The first studies to demonstrate the ability of thermographic phosphors to measure the temperature of individual droplets were presented by Omrane et al. (2004a, b, c). In the study of Omrane et al. (2004a), measurements are performed on water droplets on the order of one millimeter in diameter falling from a heated tank into air at room temperature. Both lifetime and two-line methods were applied using, respectively, $\mathrm{La}_{2} \mathrm{O}_{2} \mathrm{~S}: \mathrm{Eu}$ and $\mathrm{Mg}_{4} \mathrm{FGeO}_{6}: \mathrm{Mn}$. Relatively high temperature sensitivities are reported for both methods but for the highest accuracy, it was shown that the temporal method should be preferred, as the time decay of some thermographic phosphors like $\mathrm{La}_{2} \mathrm{O}_{2} \mathrm{~S}$ :Eu can undergo marked variations over a specific range of temperatures. More about the thermal sensitivities of lifetimes and two-line intensity ratios of some phosphors emitting in the UV/blue spectral range can be found in the rather comprehensive surveys by Särner et al. (2008a). In Omrane et al. (2004b), the time decay of $\mathrm{La}_{2} \mathrm{O}_{2} \mathrm{~S}: \mathrm{Eu}$ was used to measure the temperature of water/acetone droplets suspended in an acoustic levitator. Inorganic phosphors consist of small solid particles and have luminescence properties which are not influenced by the composition of the liquid in which they are dispersed. This is clearly an advantage over molecular tracers whose emissions generally depend upon their chemical environment. Omrane et al. (2004c) extended lifetime measurements to imaging. They used a high-speed camera containing seven independent intensified CCD detectors and a pyramidal beam splitter to subdivide the incoming light to the detectors. For accurate measurements, the time gates of the detectors and the time delay between them needed to be adjusted carefully. This can be an issue when working with high temperature gradients. Furthermore, this requires prior estimation of the investigated temperatures. Measurements with this imaging technique were performed on a free falling droplet and a water spray. Due to the relatively long lifetime of the phosphor (a few tens of $\mu$ s), the spray velocity may introduce a noticeable temporal averaging during the acquisition time.

Brübach et al. (2006) used $\mathrm{Mg}_{4} \mathrm{GeO}_{5.5} \mathrm{~F}: \mathrm{Mn}$ to measure the temperature in a spray of n-dodecane droplets. In this study, an intensified CCD camera combined with an image doubler was used to detect the luminescence signal at two emission wavelengths. Figure 28 shows typical results obtained when the injection temperature in the spray nozzle was changed. To reach a reasonable signal-to-noise ratio, the signal needed to be integrated for approximately as long as the phosphor lifetime, i.e., several ms. Such a long exposure time results in a significant averaging. Using phosphors with shorter lifetimes (in the range of a few nanoseconds) should overcome this problem. Särner et al. (2008b) suggested using $\mathrm{ZnO}: \mathrm{Zn}$ and $\mathrm{ZnO}: \mathrm{Ga}$ which have very short-lived emission lines in the UV. Moreover, these lines are significantly blue shifted as the temperature increases which introduces the possibility of using intensity ratios. Measurements with these phosphors were demonstrated in the case of methanol droplets falling individually in a Bunsen flame. Combined temperature and velocity measurements were also demonstrated by Omrane et al. (2008). In this study, the temperature of a hot air flow is determined using $\mathrm{Mg}_{4} \mathrm{FGeO}_{6}: \mathrm{Mn}$ phosphors and the two-line emission method, while the same phosphor particles also act as tracers for $2 \mathrm{D}$ velocity measurements using PIV. Although droplets are not considered in this study, perspectives can be seen in many situations including sprays.

\subsection{Molecular tagging thermometry}

Laser-induced phosphorescence (LIP) has not been used as commonly as LIF in flow diagnostics. This is mainly related to the fact that long-lived excited states largely suffer from quenching by oxygen. LIP was essentially limited to diagnostics where it offers some advantages over LIF, such as molecular tagging velocimetry (MTV). In this technique, the long lifetime of the phosphorescent molecules means their displacement between two consecutive images can be visualized to determine their velocity (Koochesfahani and Nocera 2007). Advanced applications of MTV relies on supramolecules which are almost unquenchable by oxygen, in particular the ternary complex 1-BrNp•G $\beta-\mathrm{CD} \bullet \mathrm{R}-\mathrm{OH} \quad(1-\mathrm{BrNp}$ : 1-bromonapthtalene, G $\beta$-CD: glucosyl- $\beta$-cyclodextrin, $\mathrm{R}-\mathrm{OH}$ : an alcohol) 

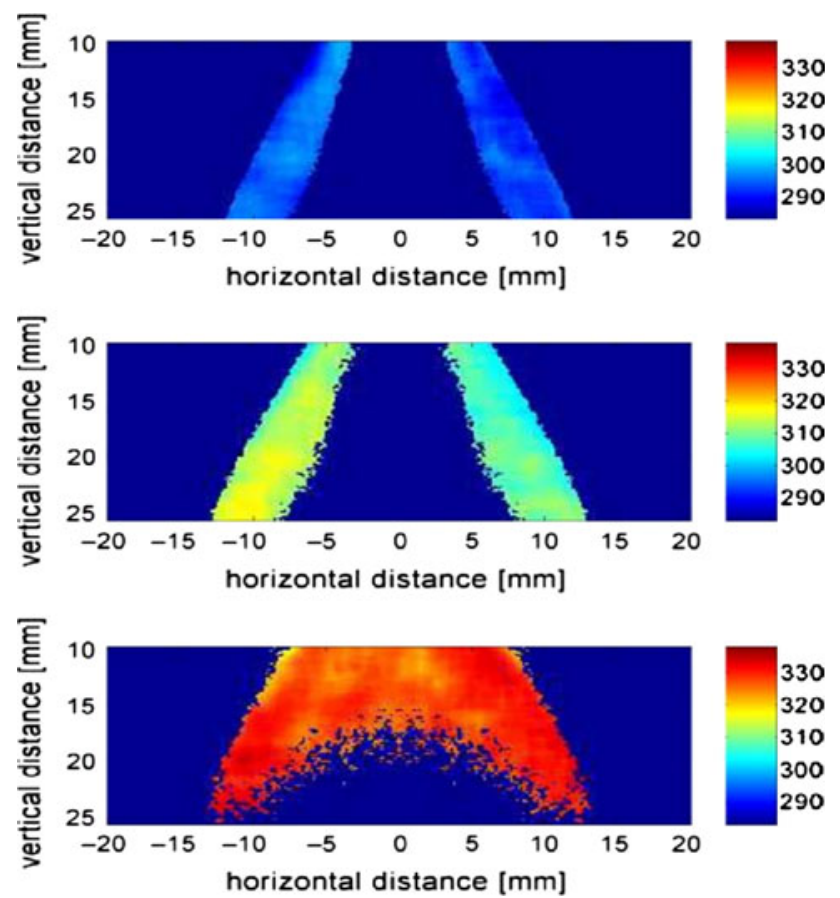

Fig. 28 Temperature map $[\mathrm{K}]$ in a spray of a n-dodecane droplets averaged over 150 single-shots for initial liquid phase temperatures of $T=296 \mathrm{~K}$ (upper plot), $T=343 \mathrm{~K}$ (central plot) and $T=393 \mathrm{~K}$ (lower plot). The point of origin is corresponding to the outlet of the nozzle. Reprinted from Brübach et al. (2006)

introduced by Gendrich et al. (1997). The emission of the latter also proved to be temperature dependent (Thomson and Maynes 2001). The derived measurement method called molecular tagging thermometry (MTT) originally suffered from limitations linked to it relying on the signal intensity of a single band. Hu and Koochesfahani (2003) developed a lifetime-based version of the MTT technique. The lifetime is determined by the intensity ratio of the tagged molecules between two consecutive images. To accurately calculate the ratio, interrogation windows are changed between the images to follow the displacement of the tagged molecules which are transported by the flow. The sensitivity of the method is also adjusted by changing the time delay between the images and between the laser pulse and the first image within the pair (Hu et al. 2006). Combined temperature and velocity measurements have been reported for various kinds of liquid flows, including pulsed jet flows (Hu and Koochesfahani 2002) and flows in the wake region of a heated cylinder ( $\mathrm{Hu}$ and Koochesfahani 2006). Recently, MTT was used to measure the temperature of sessile droplets which were frozen on a cooled plate (Hu and Jin 2010; Jin and $\mathrm{Hu} 2010$ ). In this application, displacements of the tagged molecules due to a Marangoni flow are negligible within the phosphorescence lifetime which means interrogation windows do not need to be changed within the pairs of consecutives images.

\subsection{Thermochromic liquid crystal}

Liquid crystals are highly anisotropic fluids which exist between the boundaries of the solid phase and the conventional, isotropic liquid phase. The temperature visualization is based on the property of some cholesteric and chiral-nematic liquid crystal materials to refract light of selected wavelength as a function of the temperature. The color change for the TLC ranges from clear at ambient temperature through red as temperature increases and then to yellow, green, blue and violet before turning colorless (isotropic) again at a higher temperature (Abdullah et al. 2010; Dabiri 2009). The color of light refracted by TLCs depends both on temperature and on the observation angle. Therefore, it is important that the investigated flow is illuminated by a collimated light source and observed by a camera from a fixed direction. The hue representing the dominant wavelength of the color can be obtained by a RGB analysis of the images. The hue is directly dependent on the TLCs temperature, although the color-temperature relationship is generally strongly nonlinear. The technique called digital particle image thermometry was applied in many liquid flow configurations (Dabiri 2009; Dabiri and Gharib 1991). Dispersing the liquid crystal material into the liquid makes it into a classical tracer used for flow visualization. Suspensions of thermochromic liquid crystals have been used to investigate simultaneously the temperature and velocity fields, liquid crystal particles acting as tracers for PIV measurements (Dabiri and Gharib 1996; Hiller et al. 1993; Park et al. 2001).

The use of liquid crystal thermography as a means to measure droplet temperature was reported in many references (Hu et al. 1994; Mochizuki et al. 1999; Nozaki et al. 1995; Peterson et al. 1995; Richards and Richards 1997, 1998; Treuner et al. 1995). In these studies, drop sizes were of the order of a few millimeters, while micro-encapsulated liquid crystal typically ranges to a few tens of microns and their response time is a few ms. In Richards and Richards (1998), TLC thermometry was applied to the investigation of the transient cooling of water droplets suspended at the tip of a capillary tube in dry air. In Nozaki et al. (1995) and Mochizuki et al. (1999), the technique is employed to measure the temperature of water drops flowing into an immiscible, transparent liquid medium made of silicone oil.

\subsection{Infrared thermometry and thermal imaging (IRT)}

Infrared thermal radiations have been used in a variety of droplet studies to measure the temperature mainly through thermal imaging. A particular issue with IRT methods is to correctly interpret the infrared information according to the radiative properties of the liquid. In almost all the studies, 
the spectral range of detection is selected in a region where the liquid absorbs strongly because this allows the penetration depth of infrared light to be very small in comparison with droplet size. In this condition, the observed temperature can be safely interpreted as being the surface temperature with very little contribution made by the liquid immediately beneath the surface. Therefore, the emissivity of the liquid surface is the only parameter required in order to deduce the temperature from the infrared radiance. An incorrect integral emissivity value within the spectral region of the IR camera can be a major source of measurement error. In the absence of available information, a calibration may be required to determine the value of the emissivity. Tuckermann et al. (2005) performed simultaneous measurements of the temperature of acoustically levitated droplets using a thermocouple and an IR detection system. The emissivity was adjusted to obtain the best temperature correlations between the measurements. Brutin et al. $(2010,2011)$ considered the case of semi-transparent droplets in a study of sessile droplets deposited on a flat heated surface. The authors drew attention to the fact that measurements obtained with different semi-transparent liquids correspond to different depths of penetration. The global emissivity which is deduced from the transmittivity of the liquid increases exponentially with the liquid thickness.

The effect of thermal radiations reflected from the object being measured has rarely been considered even when the droplet temperature is near the ambient temperature. Naudin et al. (1995a, b) presented an inverse model which accounts for the reflectivity of the droplet surface. In these studies, the infrared radiation emitted by a chain of monodisperse droplets was compared with that of a black body placed behind the droplets. The measurement protocol consisted of adjusting the black body temperature to produce the same radiance as the droplets when the latter are not in the detector's field of view. This approach was used to study droplets placed in high temperature ambiances like droplets flowing in flames (Naudin et al. 1995a; Ravel et al. 1997) or impacting onto a heated surface (Amiel et al. 2001). Measurements of surface temperature using this method were combined with volume average temperature measurements using $2 \mathrm{cLIF}$ thermometry by Castanet et al. (2003a).

Among all the applications of IRT to probe the droplet temperature, a significant number are also related to droplets suspended by acoustic levitation. These studies deal with evaporating droplets made of pure liquids (Tuckermann et al. 2002; Wulsten and Lee 2008), evaporating droplets made of complex mixtures (Saha et al. 2010b; Tuckermann et al. 2005) and evaporating droplets undergoing a chemical transformation and/or a change of phase (Ip et al. 2008; Liu et al. 2010; Saha et al. 2010a). In Savino and Fico (2004), drops are attached to the tip of a capillary tube and heated in a hot air stream. The use of IRT was also demonstrated for the investigation of thermocapillary convection within droplets resting on a heated solid surface (Brutin et al. 2010, 2011; Ganzevles and Van Der Geld 1998; Sefiane et al. 2008). Infrared thermography can be used to measure the occurrence of temperature gradients on the droplet surface (Ganzevles and Van Der Geld 1998) and also makes it possible to visualize cellular patterns driven by internal convection and spontaneous evaporation (Fig. 29) as reported by Brutin et al. (2010, 2011) and Sefiane et al. (2008).

\section{Conclusion and outlook}

This paper gives an overview of the optical techniques which can be used to characterize the temperature and chemical composition of droplets. Characterizing droplets is extremely important particularly for the energy sector (liquid fuel injectors, heat management systems with spray cooling) and the process industry (sprays drying or cooling). In industrial applications, droplets display complex

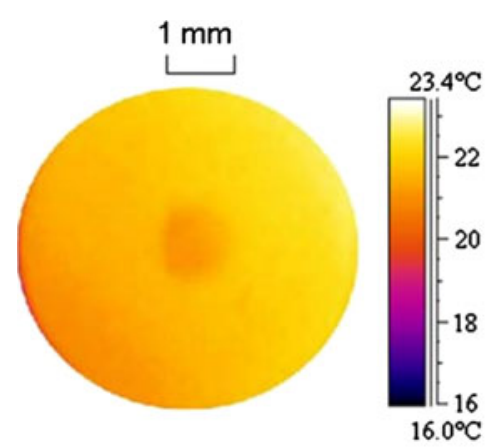

(a)

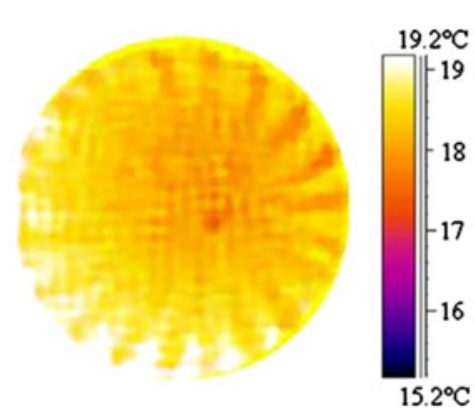

(b)

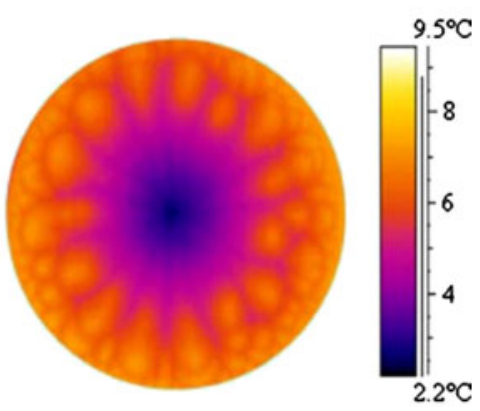

(c)

Fig. 29 Patterns observed in sessile drops of water, methanol and FC-72 refrigerant evaporating on silicon substrates, shown in panels (a-c). Reprinted from Sefiane et al. (2008) 
types of behavior: They may be strongly deformed or randomly orientated, their components may exhibit different rates of evaporation or they may also contain several phases (solid content or immiscible liquid layers). Despite the rather wide variety of techniques reviewed in this article, the requirements for measuring the temperature and composition of droplets in these complex situations are still not entirely fulfilled. Several issues remain and these represent challenges for future research work. The techniques reviewed (especially those depending on inelastic light scattering) provide indirect measurements, which means that these techniques require calibrations of the signal (intensity, shape of spectral lines, lifetime...) as a function of the parameters of interest for quantitative measurements. Calibrations need to be performed at conditions close to that of the real measurements and any extrapolating beyond these known signal conditions should be carried out with caution. Some methods (for example Rainbow refractometry or molecular LIF) are sensitive to both temperature and composition and therefore have to be used in experimental conditions where only one of the parameters varies or alternatively by combining them with another method. Despite progress in the recent years, several techniques have only been demonstrated in very simple conditions (single drop in levitation, monodisperse droplet lines.). Further research is needed before they can be applied in real spray conditions. All the presented methods have strengths and weaknesses, knowledge of which is required before using them for a specific application.

Methods based on elastic light scattering do not require additives and are relatively easy to implement. They provide a measurement of the liquid refractive index which is an intrinsic property of the liquid related to the temperature and the composition. However, measurements require a very accurate determination of the angular position of the rainbow, and signal inversion is very sensitive to refractive index gradients and to the shape and orientation of the droplets.

Raman scattering is mainly used to determine the chemical composition of the droplets by analyzing the spectral shape of specific scattering molecules. Temperature can also be determined by monitoring the spectral shift of the $\mathrm{OH}$ spectral band, when chemical composition does not vary. Spontaneous Raman spectroscopy mainly suffers from a very weak signal level which means using powerful and highly focused laser beams. If space resolved measurements can be achieved, time averaging is almost unavoidable due to the low signal. Stimulated Raman spectroscopy takes advantage of the amplification of the incident wave and the Raman signal in whispering gallery modes which gives a higher signal level and high sensitivity to composition change.
Methods based on fluorescence and phosphorescence are efficient for measuring droplet temperature and to some extent chemical composition. Approaches based on intensity ratios are preferred for temperature measurements because temperature is not the only factor which affects the signal. The addition of fluorophores is generally needed which can affect some aspects of the investigated system. The emission of molecular tracers may be sensitive to their chemical environment (chemical composition, $\mathrm{pH}$, dissolved oxygen, etc.). Therefore, their use requires extreme care and sometimes heavy calibration procedures. This problem does not exist with thermographic phosphors and mixing them with the liquid can be enhanced using nanophosphor particles being developed today. A promising alternative to intensity-based measurements is the determination of fluorescent lifetime, an intrinsic property of the emitters depending on the temperature and the chemical environment but which is not influenced by droplet morphology or the extension of the measurement volume. Applications of fluorescence lifetime to droplets are still very limited because they require fast detection devices and ultra-short laser pulses. It is likely that lifetime measurement systems will become more affordable in the future. Application to droplets could largely benefit from the wide use of lifetime measurements in cellular biology.

\section{References}

Abdullah N, Abu Talib AR, Jaafar AA, Mohd Salleh MA, Chong WT (2010) The basics and issues of thermochromic liquid crystal calibrations. Exp Thermal Fluid Sci 34:1089-1121

Abramzon B, Sirignano WA (1989) Droplet vaporization model for spray combustion calculations. Int $\mathrm{J}$ Heat Mass Transf 32: $1605-1618$

Airy G (1838) On the intensity of light in the neighbourhood of a caustic. Trans Camb Phyl Soc 6:397-403

Aldén M, Omrane A, Richter M, Särner G (2011) Thermographic phosphors for thermometry: a survey of combustion applications. Prog Energy Combust Sci 37:422-461

Allison SW, Gillies GT (1997) Remote thermometry with thermographic phosphors: Instrumentation and applications. Rev Sci Instrum 68:2615-2650

Amiel C, Le Clercq P, Ravel O, Lavergne G, Berthoumieu P, Farre J (2001) Use of an infrared detector to analyze the temperature evolution of a droplet impacting on a heating wall. In: Rozlosnik AE, Dinwiddie, RB(eds) Thermosense xxiii, vol 4360. pp 13-20

Anders K, Roth N, Frohn A (1996) Influence of refractive index gradients within droplets on rainbow position and implications for rainbow refractometry. Part Part Syst Charact 13:125-129

Anderson A (1971) The Raman effect: principles, vol 1. Marcel Dekker, New York

Anderson A (1973) The Raman effect: applications, vol 2. Marcel Dekker, New York

Ashkin A (1970) Acceleration and trapping of particles by radiation pressure. Phys Rev Lett 24:156-159

Bai FL, Melton LA (1997) High-temperature, oxygen-resistant molecular fluorescence thermometers. Anglais 51:1276-1280 
Biswas A, Latifi H, Armstrong RL, Pinnick RG (1989) Doubleresonance stimulated raman-scattering from optically levitated glycerol droplets. Phys Rev A 40:7413-7416

Blatt E, Treloar FE, Ghiggino KP, Gilbert RG (1981) Viscosity and temperature dependence of fluorescence lifetimes of anthracene and 9-methylanthracene. J Phys Chem 85:2810-2816

Bonin D, Saengkaew S, Han YP, Grehan G (2007) On non-sphericity detection in standard rainbow refractometry. In: 8th International congress on optical particle characterization, Graz, Austria

Brübach J, Patt A, Dreizler A (2006) Spray thermometry using thermographic phosphors. Appl Phys B Lasers Opt 83:499-502

Bruchhausen M, Guillard F, Lemoine F (2004) Instantaneous measurement of two-dimensional temperature distributions by means of two-color planar laser induced fluorescence (plif). Exp Fluids 38:123-131

Bruchhausen M, Delconte A, Blondel D, Lemoine F (2006) Temperature measurements in polydisperse sprays by means of laserinduced fluorescence (lif) on three spectral bands. At Sprays 16:599-614

Brutin D, Zhu ZQ, Rahli O, Xie JC, Liu QS, Tadrist L (2010) Evaporation of ethanol drops on a heated substrate under microgravity conditions. Microgravity Sci Technol 22:387-395

Brutin D, Sobac B, Rigollet F, Le Niliot C (2011) Infrared visualization of thermal motion inside a sessile drop deposited onto a heated surface. Exp Thermal Fluid Sci 35:521-530

Buehler MF, Allen TM, Davis EJ (1991) Microparticle Raman spectroscopy of multicomponent aerosols. J Colloid Interface Sci 146:79-89

Carls JC, Moncivais G, Brock JR (1990) Time-resolved Raman spectroscopy from reacting optically levitated microdroplets. Appl Opt 29:2913-2918

Casadevall i Solvas X, Srisa-Art M, DeMello AJ, Edel JB (2010) Mapping of fluidic mixing in microdroplets with 1 micros time resolution using fluorescence lifetime imaging. Anal Chem $82: 3950-3956$

Casadevall i Solvas X, Niu X, Leeper K, Cho S, Chang S-I, Edel JB, deMello AJ (2011) Fluorescence detection methods for microfluidic droplet platforms. J Vis Exp e3437

Castanet G, Lavieille P, Lemoine F, Lebouché M, Atthasit A, Biscos Y, Lavergne G (2002) Energetic budget on an evaporating monodisperse droplet stream using combined optical methods: Evaluation of the convective heat transfer. Int $\mathbf{J}$ Heat Mass Transf 45:5053-5067

Castanet G, Delconte A, Lavieille P, Lemoine F, Amiel C, Berthoumieu P, Lavergne G (2003a) Simultaneous measurements of mean and surface temperature of evaporating moving droplets using combined two colors laser-induced fluorescence and infrared thermometry. Extension to the measurement of the temperature distribution within the droplets. 9th International Congress on Liquid Atomization and Spray Systems, Sorrento, Italy

Castanet G, Lavieille P, Lebouché M, Lemoine F (2003b) Measurement of the temperature distribution within monodisperse combusting droplets in linear streams using two-color laserinduced fluorescence. Exp Fluids 35:563-571

Castanet G, Delconte A, Lemoine F, Mees L, Gréhan G (2005a) Evaluation of temperature gradients within combusting droplets in linear stream using two colors laser-induced fluorescence. Exp Fluids 39:431-440

Castanet G, Lavieille P, Lebouché M, Lemoine F (2005b) Experimental and theoretical investigation of the heating of combusting droplets in a linear stream. Combust Sci Technol 177: 2395-2422

Castanet G, Lebouche M, Lemoine F (2005c) Heat and mass transfer of combusting monodisperse droplets in a linear stream. Int $\mathbf{J}$ Heat Mass Transf 48:3261-3275
Castanet G, Maqua C, Orain M, Grisch F, Lemoine F (2007) Investigation of heat and mass transfer between the two phases of an evaporating droplet stream using laser-induced fluorescence techniques: Comparison with modeling. Int $\mathrm{J}$ Heat Mass Transf 50:3670-3683

Castanet G, Lienart T, Lemoine F (2009) Dynamics and temperature of droplets impacting onto a heated wall. Int J Heat Mass Transf 52:670-679

Castanet G, Frackowiak B, Tropea C, Lemoine F (2011a) Heat convection within evaporating droplets in strong aerodynamic interactions. Int J Heat Mass Transf 54:3267-3276

Castanet G, Labergue A, Lemoine F (2011b) Internal temperature distributions of interacting and vaporizing droplets. Int J Therm Sci 50:1181-1190

Chen G, Serpengüzel A, Chang RK, Acker WP (1993) Relative evaporation of droplets in a segmented stream determined by droplet cavity fluorescence peak shifts. In: SPIE conference on laser applications in combustion and combustion diagnostics. Los Angeles, pp. 200-208

Chen G, Mazumder MM, Chang RK, Swindal JC, Acker WP (1996) Laser diagnostics for droplet characterization: Application of morphology dependent resonances. Prog Energy Combust Sci 22:163-188

Coppeta J, Rogers C (1998) Dual emission laser induced fluorescence for direct planar scalar behavior measurements. Exp Fluids 25:1-15

Crimaldi JP (2008) Planar laser induced fluorescence in aqueous flows. Exp Fluids 44:851-863

Dabiri D (2009) Digital particle image thermometry/velocimetry: a review. Exp Fluids 46:191-241

Dabiri D, Gharib M (1991) Digital particle image thermometry: the method and implementation. Exp Fluids 11:77-86

Dabiri D, Gharib M (1996) The effects of forced boundary conditions on flow within a cubic cavity using digital particle image thermometry and velocimetry (dpitv). Exp Thermal Fluid Sci 13:349-363

Damaschke N (2003) Light scattering theories and their use for single particle characterization. In: $\mathrm{PhD}$ : Technische Universitat Darmstadt

Davis EJ, Buehler MF, Ward TL (1990) The double-ring electrodynamic balance for microparticle characterization. Rev Sci Instrum 61:1281-1288

Depredurand V, Miron P, Labergue A, Wolff M, Castanet G, Lemoine F (2008) A temperature-sensitive tracer suitable for two-colour laser-induced fluorescence thermometry applied to evaporating fuel droplets. Meas Sci Technol 19:105403

Depredurand V, Delconte A, Lemoine F (2011) Combined pda and lif applied to size-temperature correlations measurements in a heated spray. Exp Fluids 50:561-571

Deprédurand V, Castanet G, Lemoine F (2010) Heat and mass transfer in evaporating droplets in interaction: Influence of the fuel. Int J Heat Mass Transf 53:3495-3502

Desantes JM, Pastor JV, Pastor JM, Julia JE (2005) Limitations on the use of the planar laser induced exciplex fluorescence technique in diesel sprays. Fuel 84:2301-2315

Domann R, Hardalupas Y (2001) Spatial distribution of fluorescence intensity within large droplets and its dependence on dye concentration. Appl Opt 40:3586-3597

Domann R, Hardalupas Y, Jones AR (2002) A study of the influence of absorption on the spatial distribution of fluorescence intensity within large droplets using mie theory, geometrical optics and imaging experiments. Meas Sci Technol 13:280-291

Dunand P, Castanet G, Lemoine F (2012) A two-color planar lif technique to map the temperature of droplets impinging onto a heated wall. Exp Fluids 52:843-856 
Dunand P, Castanet G, Gradeck M, Lemoine F, Maillet D (2013) Heat transfer of droplets impinging onto a wall above the leidenfrost temperature. Comptes Rendus Mécanique 341:75-87

Duwel I, Ge HW, Kronemayer H, Dibble R, Gutheil E, Schulz C, Wolfrum J (2007) Experimental and numerical characterization of a turbulent spray flame. Proc Combust Inst 31:2247-2255

Eckbreth AC, Bonczyk PA, Verdieck JF (1979) Combustion diagnostics by laser Raman and fluorescence techniques. Prog Energy Combust Sci 5:253-322

Escobar S, Gonzalez JE, Rivera LA (2001) Laser-induced fluorescence temperature sensor for in-flight droplets. Exp Heat Transfer 14:119-134

Fansler TD, Drake MC, Gajdeczko B, Düwel I, Koban W, Zimmermann FP, Schulz C (2009) Quantitative liquid and vapor distribution measurements in evaporating fuel sprays using laser-induced exciplex fluorescence. Meas Sci Technol 20:125401

Felton P, Bracco F, Bardsley M (1993) On the quantitative application of exciplex fluorescence to engine sprays. SAE Technical Paper 930870

Frackowiak B, Tropea C (2010a) Fluorescence modeling of droplets intersecting a focused laser beam. Opt Lett 35:1386-1388

Frackowiak B, Tropea C (2010b) Numerical analysis of diameter influence on droplet fluorescence. Appl Opt 49:2363-2370

Fujimoto H, Choi D, Shima Y, Senda J (2002) Two-dimensional imaging of fuel-vapour concentration by use of lief technique during mixture formation process in a di diesel engine. Meas Sci Technol 13:391-400

Ganzevles FLA, Van Der Geld CWM (1998) Marangoni convection in binary drops in air cooled from below. Int J Heat Mass Transf 41:1293-1301

Gendrich CP, Koochesfahani MM, Nocera DG (1997) Molecular tagging velocimetry and other novel applications of a new phosphorescent supramolecule. Exp Fluids 23:361-372

Ghandhi JB, Felton PG, Gajdeczko BF, Bracco FV (1994) Investigation of the fuel distribution in a two-stroke engine with an airassisted injector SAE Paper 940394

Gossage HE, Melton LA (1987) Fluorescence thermometers using intramolecular exciplexes. Appl Opt 26:2256-2259

Gouesbet G, Gréhan G (2011) Generalized lorenz-mie theories, 1st edn. Springer, Berlin

Han XG, Ren KF, Wu ZS, Corbin F, Gouesbet G, Grehan G (1998) Characterization of initial disturbances in a liquid jet by rainbow sizing. Appl Opt 37:8498-8503

Han YP, Mees L, Ren KF, Gouesbet G, Wu SZ, Grehan G (2002) Scattering of light by spheroids: the far field case. Opt Commun 210:1-9

Hanlon TR, Melton LA (1992) Exciplex fluorescence thermometry of falling hexadecane droplets. J Heat Transfer 114:450-457

Heinisch C, Wills JB, Reid JP, Tschudi T, Tropea C (2009) Temperature measurement of single evaporating water droplets in a nitrogen flow using spontaneous Raman scattering. Phys Chem Chem Phys 11:9720-9728

Herzberg G (1945) Molecular spectra and molecular structure, infrared and Raman spectra of polyatomic molecules, vol 2. Van Nostrand Reinhold Corp, New York

Herzberg G (1950) Molecular spectra and molecular structure, spectra of diatomic molecules, vol 1. Van Nostrand Reinhold Corp

Hill SC, Brenner RE (1988) Morphology-dependent resonances. In: Optical effects associated with small particles. Singapore: World scientific

Hiller WJ, Koch S, Kowalewski TA, Stella F (1993) Onset of natural convection in a cube. Int J Heat Mass Transf 36:3251-3263

Homer CJ, Jiang X, Ward TL, Brinker CJ, Reid JP (2009) Measurements and simulations of the near-surface composition of evaporating ethanol-water droplets. Phys Chem Chem Phys 11:7780-7791
Hopkins RJ, Reid JP (2005) Evaporation of ethanol/water droplets: Examining the temporal evolution of droplet size, composition and temperature. J Phys Chem A 109:7923-7931

Hopkins RJ, Reid JP (2006) A comparative study of the mass and heat transfer dynamics of evaporating ethanol/water, methanol/water, and 1-propanol/water aerosol droplets. J Phys Chem B 110: 3239-3249

Hopkins RJ, Symes R, Sayer RM, Reid JP (2003) Determination of the size and composition of multicomponent ethanol/water droplets by cavity-enhanced Raman scattering. Chem Phys Lett 380:665-672

Hopkins RJ, Howle CR, Reid JP (2006) Measuring temperature gradients in evaporating multicomponent alcohol/water droplets. Phys Chem Chem Phys 8:2879-2888

Howle CR, Homer CJ, Hopkins RJ, Reid JP (2007) Probing the evaporation of ternary ethanol-methanol-water droplets by cavity enhanced Raman scattering. Phys Chem Chem Phys 9: $5344-5352$

Hu H, Jin Z (2010) An icing physics study by using lifetime-based molecular tagging thermometry technique. Int $\mathbf{J}$ Multiph Flow $36: 672-681$

$\mathrm{Hu} \mathrm{H}$, Koochesfahani M (2002) A novel technique for quantitative temperature mapping in liquid by measuring the lifetime of laser induced phosphorescence. Proceedings of 10th International Symposium on Flow Visualization, Kyoto, Japan

$\mathrm{Hu} \mathrm{H}$, Koochesfahani MM (2003) A novel technique for quantitative temperature mapping in liquid by measuring the lifetime of laser induced phosphorescence. J Vis 6:143-153

Hu H, Koochesfahani M (2006) Molecular tagging velocimetry and thermometry and its application to the wake of a heated circular cylinder. Meas Sci Technol 17:1269

Hu S, Richards RF, Richards CD (1994) Thermography of atomized droplets in flight using thermochromic liquid crystals. The seventh annual ILASS-Americas conference on liquid atomization and spray systems

$\mathrm{Hu} \mathrm{H}$, Koochesfahani M, Lum C (2006) Molecular tagging thermometry with adjustable temperature sensitivity. Exp Fluids 40:753-763

Ip LT, Baxter LL, Mackrory AJ, Tree DR (2008) Surface temperature and time-dependent measurements of black liquor droplet combustion. AIChE J 54:1926-1931

Jin Z, Hu H (2010) Icing process of small water droplets impinging onto a frozen cold plate. J Thermophys Heat Transfer 24:841-845

Kadota T, Taniguchi Y, Kadowaki K (1991) Exciplex method for remote probing of fuel droplet temperature. JSME Int J Ser II $34: 242-247$

Kim JH (2007) Spray cooling heat transfer: the state of the art. Int J Heat Fluid Flow 28:753-767

Kim HJ, Kihm KD, Allen JS (2003) Examination of ratiometric laser induced fluorescence thermometry for microscale spatial measurement resolution. Int J Heat Mass Transf 46:3967-3974

König G, Anders K, Frohn A (1986) A new light-scattering technique to measure the diameter of periodically generated moving droplets. J Aerosol Sci 17:157-167

Koochesfahani MM, Nocera DG (2007) Molecular tagging velocimetry, chapter 5.4. In: Handbook of experimental fluid dynamics. Springer, Heidelberg

Labergue A, Deprédurand V, Delconte A, Castanet G, Lemoine F (2010) New insight into two-color lif thermometry applied to temperature measurements of droplets. Exp Fluids 49:547-556

Labergue A, Delconte A, Castanet G, Lemoine F (2012a) Study of the droplet size effect coupled with the laser light scattering in sprays for two-color lif thermometry measurements. Exp Fluids 52:1121-1132

Labergue A, Delconte A, Lemoine F (2012b) Three-color lif thermometry applied to the mixing of two non-isothermal sprays 
16th International Symposium on Applications of Laser Techniques to Fluid Mechanics, lisbon

Lakowicz JR (2006) Principles of fluorescence spectroscopy, 3rd edn. Springer, New York

Lakowicz JR, Szmacinski H (1993) Fluorescence lifetime-based sensing of ph, ca $2+, \mathrm{k}+$ and glucose. Sens Actuators B Chem 11:133-143

Laurent C, Biscos Y, Doué N, Maqua C, Lemoine F, Grehan G, Lavergne $G$ (2006) Thermal gradient determination inside vaporizing droplets by combining rainbow and laser induced fluorescence measurements. In: Proceedings of ASME Fluids Engineering Division Summer Meeting 2006, FEDSM2006, Miami, FL

Lavieille P, Lemoine F, Lavergne G, Virepinte JF, Lebouché M (2000) Temperature measurements on droplets in monodisperse stream using laser-induced fluorescence. Exp Fluids 29:429-437

Lavieille P, Lemoine F, Lavergne G, Lebouché M (2001) Evaporating and combusting droplet temperature measurements using twocolor laser-induced fluorescence. Exp Fluids 31:45-55

Lavieille P, Lemoine F, Lebouché M (2002) Investigation on temperature of evaporating droplets in linear stream using twocolor laser-induced fluorescence. Combust Sci Technol 174:117-142

Lavieille P, Delconte A, Blondel D, Lebouché M, Lemoine F (2004) Non-intrusive temperature measurements using three-color laserinduced fluorescence. Exp Fluids 36:706-716

Lee RL (1998) Mie theory, airy theory, and the natural rainbow. Appl Opt 37:1506-1519

Lemoine F, Wolff M, Lebouche M (1996) Simultaneous concentration and velocity measurements using combined laser-induced fluorescence and laser doppler velocimetry: Application to turbulent transport. Exp Fluids 20:319-327

Lemoine F, Antoine Y, Wolff M, Lebouche M (1999) Simultaneous temperature and $2 \mathrm{~d}$ velocity measurements in a turbulent heated jet using combined laser-induced fluorescence and lda. Exp Fluids 26:315-323

Lin HB, Eversole JD, Campillo AJ (1990) Identification of morphology dependent resonances in stimulated raman-scattering from microdroplets. Opt Commun 77:407-410

Liu L, Bi QC, Terekhov VI, Shishkin NE (2010) Experimental investigation evaporation of liquid mixture droplets during depressurization into air stream. In: AIP conference proceedings, Xian

Lu QZ, Melton LA (2000) Measurement of transient temperature field within a falling droplet. AIAA J 38:95-101

Maqua C, Castanet G, Lemoine F, Doué N, Lavergne G (2006) Temperature measurements of binary droplets using three-color laser-induced fluorescence. Exp Fluids 40:786-797

Maqua C, Depredurand V, Castanet G, Wolff M, Lemoine F (2007) Composition measurement of bicomponent droplets using laserinduced fluorescence of acetone. Exp Fluids 43:979-992

Massoli P (1997) Temperature and size of droplets inferred by light scattering methods: A theoretical analysis of the influence of internal inhomogeneities. In: 13th Annual Conference on Liquid Atomization and Spray Systems ILASS- Europe, Florence

Massoli P (1998) Rainbow refractometry applied to radially inhomogeneous spheres: The critical case of evaporating droplets. Appl Opt 37:3227-3235

Massoli P, Beretta F, D'Alessio A, Lazzaro M (1993) Temperature and size of single transparent droplets by light scattering in the forward and rainbow regions. Appl Opt 32:3295-3301

Matsumoto K, Fujii T, Suzuki K, Segawa D, Kadota T (1999) Laserinduced fluorescence for the non-intrusive diagnostics of a fuel droplet burning under microgravity in a drop shaft. Meas Sci Technol 10:853
Mazumder M, Gang Chen PJ, Kindlmann PJ, Chang RK (1995a) Droplet-temperature determination using thermochromic additives. In: CLEO'95, Baltimore, USA

Mazumder MM, Chen G, Kindlmann PJ, Chang RK, Gillespie JB (1995b) Temperature-dependent wavelength shifts of dye lasing in microdroplets with a thermochromic additive. Opt Lett 20:1668

McGlashen ML, Davis KL, Morris MD (1990) Surface-enhanced raman-scattering of dopamine at polymer-coated silver electrodes. Anal Chem 62:846-849

Megahed M (1993a) Estimation of the potential of a fluorescnce thermometer for diesel spray studies. Appl Opt 32:4790-4796

Megahed M (1993b) First measurements of the liquid phase temperature in diesel sprays. SAE technical paper series:930969

Melton LA (1983) Spectrally separated fluorescence emissions for diesel fuel droplets and vapor. Appl Opt 22:2224-2226

Min SL, Gomez A (1996) High-resolution size measurement of single spherical particles with a fast fourier transform of the angular scattering intensity. Appl Opt 35:4919-4926

Mochizuki T, Nozaki T, Mori YH, Kaji N (1999) Heat transfer to liquid drops passing through an immiscible liquid medium between tilted parallel-plate electrodes. Int J Heat Mass Transf 42:3113-3129

Moebius W (1910) Zur theorie des regenboges und ihren experimentellen prufung. Ann Phys 33:1493-1558

Müller T, Beushausen V, Hentschel W (2000a) 1d spontaneous Raman scattering for comprehensive $2 \mathrm{~d}$ spray characterization. In: 8th International Conference on Liquid Atomization and Spray Systems, Pasadena, USA

Müller T, Grünefeld G, Beushausen V (2000b) High-precision measurement of the temperature of methanol and ethanol droplets using spontaneous Raman scattering. Appl Phys B Lasers Opt 70:155-158

Murray AM, Melton LA (1985) Fluorescence methods for determination of temperature in fuel sprays. Appl Opt 24:2783-2787

Naudin N, Farre J, Bissieres D, Lavergne G (1995a) Ir instrumentation dedicated to the experimental validation of evaporating and burning droplets' temperature models. Vol. 2552

Naudin N, Farre JA, Lavergne G (1995b) Infrared system for methanol-droplets temperature measurement on a monodisperse jet. Proc Soc Photo Opt Instrum Eng 2473:338-345

Nozaki T, Mochizuki T, Kaji N, Mori YH (1995) Application of liquid-crystal thermometry to drop temperature measurements. Exp Fluids 18:137-144

Nussenzveig HM (1969) High-frequency scattering by a transparent sphere.2. Theory of rainbow and glory. J Math Phys 10:125-176

Omrane A, Juhlin G, Ossler F, Aldén M (2004a) Temperature measurements of single droplets by use of laser-induced phosphorescence. Appl Opt 43:3523-3529

Omrane A, Santesson S, Alden M, Nilsson S (2004b) Laser techniques in acoustically levitated micro droplets. Lab Chip 4:287-291

Omrane A, Särner G, Aldén M (2004c) 2d-temperature imaging of single droplets and sprays using thermographic phosphors. Appl Phys B Lasers Opt 79:431-434

Omrane A, Petersson P, Aldén M, Linne MA (2008) Simultaneous 2d flow velocity and gas temperature measurements using thermographic phosphors. Appl Phys B 92:99-102

Owen JF, Chang RK, Barber PW (1982) Morphology-dependent resonances in raman-scattering, fluorescence emission, and elastic-scattering from microparticles. Aerosol Sci Technol $1: 293-302$

Panao MRO, Moreira ALN (2009) Intermittent spray cooling: A new technology for controlling surface temperature. Int J Heat Fluid Flow 30:117-130 
Park HG, Dabiri D, Gharib M (2001) Digital particle image velocimetry/thermometry and application to the wake of a heated circular cylinder. Exp Fluids 30:327-338

Peterson D, Hu S, Richards CD, Richards RF (1995) The measurement of droplet temperature using thermochromic liquid crystals. ASME HDT 308:39-46

Ravel O, Naudin N, Adam O, Virepinte JF, Farre J, Lavergne G (1997) Infrared temperature measurement of burning droplet. Vol. 3108

Reid JP, Meresman H, Mitchem L, Symes R (2007) Spectroscopic studies of the size and composition of single aerosol droplets. Int Rev Phys Chem 26:139-192

Richards CD, Richards RF (1997) Convective cooling of a suspended water droplet. J Heat Transf 119:208

Richards CD, Richards RF (1998) Transient temperature measurements in a convectively cooled droplet. Exp Fluids 25:392-400

Roth N, Anders K, Frohn A (1988) Simultaneous measurement of temperature and size of droplet in the micrometer range. In: 7th International congress on optical methods in flow and particle diagnostics ICALEO 88, Sunnyvale, vol 67, pp 294-304

Roth N, Anders K, Frohn A (1991) Refractive-index measurements for the correction of particle sizing methods. Appl Opt 30:4960-4965

Roth N, Anders K, Frohn A (1992) Simultaneous determination of refractive index and droplet size using mie theorie. In: 6th International symposium on applications of laser techniques to fluid mechanics, Lisbon, Portugal

Saengkaew S, Charinpanitkul T, Vanisri H, Tanthapanichakoon W, Mees L, Gouesbet G, Grehan G (2006) Rainbow refractrometry: on the validity domain of airy's and nussenzveig's theories. Opt Commun 259:7-13

Saengkaew S, Charinpanitkul T, Vanisri H, Tanthapanichakoon W, Biscos Y, Garcia N, Lavergne G, Mees L, Gouesbet G, Grehan G (2007) Rainbow refractrometry on particles with radial refractive index gradients. Exp Fluids 43:595-601

Saengkaew S, Godard G, Blaisot J, Gréhan G (2009) Experimental analysis of global rainbow technique: sensitivity of temperature and size distribution measurements to non-spherical droplets. Exp Fluids 47:839-848

Saengkaew S, Charinpanikul T, Laurent C, Biscos Y, Lavergne G, Gouesbet G, Gréhan G (2010) Processing of individual rainbow signals. Exp Fluids 48:111-119

Saengkaew S, Ouboukhil M, Estel L, Grehan G (2012) Coupling global rainbow technique (grt) and pda to extract the refractive index value by class of size: Application to co 2 capture by mea spray. In: International Conference on Liquid Atomization and Spray Systems, Heidelberg, Germany

Saha A, Basu S, Suryanarayana C, Kumar R (2010a) Experimental analysis of thermo-physical processes in acoustically levitated heated droplets. Int J Heat Mass Transf 53:5663-5674

Saha A, Kumar R, Basu S (2010b) Infrared thermography and numerical study of vaporization characteristics of pure and blended bio-fuel droplets. Int J Heat Mass Transf 53:3862-3873

Saharin S, Lefort B, Morin C, Chauveau C, Le Moyne L, Kafafy R (2012) Vaporization characteristics of ethanol and 1-propanol droplets at high temperatures. At Sprays 22:207-226

Sakakibara J, Adrian RJ (1999) Whole field measurement of temperature in water using two-color laser induced fluorescence. Exp Fluids 26:7-15

Sakakibara J, Adrian RJ (2004) Measurement of temperature field of a rayleigh-benard convection using two-color laser-induced fluorescence. Exp Fluids 37:331-340

Sankar S, Ibrahim K, Buermann M, Fridrich M, Bachalo W (1993) An integrated phase doppler/rainbow refractometer system for simultaneous measurement of droplet size, velocity and refractive index. In: The third international congress on optical particle sizing, pp 275-284, Yokohama, Japan
Särner G, Richter M, Aldén M (2008a) Investigations of blue emitting phosphors for thermometry. Meas Sci Technol 19:125304

Särner G, Richter M, Aldén M (2008b) Two-dimensional thermometry using temperature-induced line shifts of zno: $\mathrm{Zn}$ and zno: Ga fluorescence. Opt Lett 33:1327-1329

Savino R, Fico S (2004) Transient marangoni convection in hanging evaporating drops. Phys Fluids 16:3738-3754

Sazhin SS, Kristyadi T, Abdelghaffar WA, Heikal MR (2006) Models for fuel droplet heating and evaporation: Comparative analysis. Fuel 85:1613-1630

Sazhin SS, Elwardany A, Krutitskii PA, Castanet G, Lemoine F, Sazhina EM, Heikal MR (2010) A simplified model for bicomponent droplet heating and evaporation. Int $\mathrm{J}$ Heat Mass Transf 53:4495-4505

Sazhin SS, Elwardany AE, Krutitskii PA, Deprédurand V, Castanet G, Lemoine F, Sazhina EM, Heikal MR (2011) Multi-component droplet heating and evaporation: Numerical simulation versus experimental data. Int J Therm Sci 50:1164-1180

Schneider M, Hilerman E (1993) Rainbows and radially inhomogeneous fuel doplets in a high temperature environment. In: Third International Congress on Optical Particle Sizing, Yokohama, Japan

Schweiger G (1987) Insitu determination of the molecular composition of aerosol-particles in a monodisperse model aerosol. Part Charact 4:67-73

Schweiger G (1990) Raman-scattering on single aerosol-particles and on flowing aerosols: a review. J Aerosol Sci 21:483-509

Sefiane K, Moffat JR, Matar OK, Craster RV (2008) Self-excited hydrothermal waves in evaporating sessile drops. Appl Phys Lett 93:3

Seydel P, Blomer J, Bertling J (2006) Modeling particle formation at spray drying using population balances. Dry Technol 24:137-146

Shafii MB, Lum CL, Koochesfahani MM (2009) In situ lif temperature measurements in aqueous ammonium chloride solution during uni-directional solidification. Exp Fluids 48:651-662

Shringi DS, Shaw BD, Dwyer H (2009) Laser-induced fluorescence imaging of acetone inside evaporating and burning fuel droplets. Opt Lasers Eng 47:51-56

Stowers MA, Friedlander SK (2002) Chemical characterization of flowing polydisperse aerosols by raman spectroscopy. Aerosol Sci Technol 36:48-61

Sutton J, Fisher BT, Fleming JW (2008) A laser-induced fluorescence measurement for aqueous fluid flows with improved temperature sensitivity. Exp Fluids 45:869-881

Swindal JC, Chen G, Scheschak K, Chang RK, Jackson T (1996) Measurement of the evaporation rates of closely spaced flowing droplets by optical cavity resonances. At Sprays 6:331-351

Teh SY, Lin R, Hung LH, Lee AP (2008) Droplet microfluidics. Lab Chip 8:198-220

Thomson SL, Maynes D (2001) Spatially resolved temperature measurements in a liquid using laser induced phosphorescence. J Fluids Eng 123:293-302

Tong AY, Sirignano WA (1986) Multicomponent droplet vaporization in a high-temperature gas. Combust Flame 66:221-235

Treuner M, Rath HJ, Duda U, Siekmann J (1995) Thermocapillary flow in drops under low gravity analysed by the use of liquid crystals. Exp Fluids 19:264-273

Tropea C, Yarin AL, Foss JF (2007) Temperature measurement via absorption, light scattering and laser-induced fluorescence. In: Experimental fluid mechanics, Berlin, Heidelberg, pp 516-522

Tuckermann R, Bauerecker S, Neidhart B (2002) Evaporation rates of alkanes and alkanols from acoustically levitated drops. Anal Bioanal Chem 372:122-127

Tuckermann R, Bauerecker S, Cammenga H (2005) Ir-thermography of evaporating acoustically levitated drops. Int $\mathbf{J}$ Thermophys 26:1583-1594 
Tuckermann R, Puskar L, Zavabeti M, Sekine R, McNaughton D (2009) Chemical analysis of acoustically levitated drops by raman spectroscopy. Anal Bioanal Chem 394:1433-1441

van Beeck JP (1997) Rainbow phenomena: Development of a laserbased, non intrusive technique for measuring droplet size, temperature, velocity. Vol. PhD Thesis: Technical University of Eindhoven, Netherlands

van Beeck JP, Riethmuller ML (1994) Simultaneous determination of temperature and size of droplets from the rainbow using airy theory. In: International symposium on applications of laser techniques to fluid mechanics. Springer, Lisbon, pp. 330-339

van Beeck J, Riethmuller ML (1995) Nonintrusive measurements of temperature and size of single falling raindrops. Appl Opt 34:1633-1639

van Beeck JP, Riethmuller ML (1996) Rainbow phenomena applied to the measurement of droplet size and velocity and to the detection of nonsphericity. Appl Opt 35:2259-2266

van Beeck JP, Riethmuller ML (1998) Rainbow thermometry with a pulsed laser. In: 9th International Symposium on Applications of Laser Techniques to Fluid Mechanics, Lisbon, Portugal

van Beeck JP, Giannoulis D, Zimmer L, Riethmuller M (1999) Global rainbow thermometry for droplet-temperature measurement. Opt Lett 24:1696-1698

van Beeck J, Zimmer L, Riethmuller ML (2001) Global rainbow thermometry for mean temperature and size measurement of spray droplets. Part Part Syst Charact 18:196-204

van Beeck J, Grosges T, De Giorgi MG (2003) Global rainbow thermometry assessed by airy and lorenz-mie theories and compared with phase doppler anemometry. Appl Opt 42: 4016-4022

van de Hulst H (1981) Light scattering by small particles. New York

Vehring R, Schweiger G (1992) Optical determination of the temperature of transparent microparticles. Appl Spectrosc 46: $25-27$

Vehring R, Moritz H, Niekamp D, Schweiger G, Heinrich P (1995) Linear raman-spectroscopy on droplet chains: a new experimental-method for the analysis of fast transport processes and reactions on microparticles. Anglais 49:1215-1224

Vehring R, Aardahl CL, Schweiger G, Davis EJ (1998) The characterization of fine particles originating from an uncharged aerosol: Size dependence and detection limits for raman analysis. J Aerosol Sci 29:1045-1061

Vetrano M, van Beeck J, Riethmuller M (2004) Global rainbow thermometry: Improvements in the data inversion algorithm and validation technique in liquid-liquid suspension. Appl Opt 43:3600-3607

Vetrano MR, Simonini A, Steelant J, Lamanna G, Kamoun H, Rambaud P (2012) Flashing jet characterization by non intrusive laser based measurement technique. 16th International Symposium on Applications of Laser Techniques to Fluid Mechanics, lisbon

Walker JD (1976) Multiple rainbows from single drops of water and other liquids. Am J Phys 44:421-433

Walker DA (1987) A fluorescence technique for measurement of concentration in mixing liquids. J Phys E Sci Instrum 20:217

Walrafen GE (1967) Raman spectral studies of effects of temperature on water structure. J Chem Phys 47:114

Wells MR, Melton LA (1990) Temperature measurements of falling droplets. J Heat Transf 112:1008-1013

Wilms J, Weigand B (2006) Mass transfer in evaporating binary mixture droplets. In: International conference on liquid atomization and spary systems ICLASS, Kyoto, Japan

Wilms J, Roth N, Weigand B, Arndt S (2004) Determination of the composition of multicomponent droplets by rainbow refractometry. In: International Symposium on Appl of Laser Techniques to Fluid Mechanics

Wilms J, Gréhan G, Lavergne G (2008) Global rainbow refractometry with a selective imaging method. Part Part Syst Charact 25: $39-48$

Winter M, Melton LA (1990) Measurement of internal circulation in droplets using laser-induced fluorescence. Appl Opt 29: 4574-4577

Wolff M, Delconte A, Schmidt F, Gucher P, Lemoine F (2007) Highpressure diesel spray temperature measurements using twocolour laser-induced fluorescence. Meas Sci Technol 18: 697-706

Wu JJ, Nguyen HV, Flagan RC (1987) A method for the synthesis of submicron particles. Langmuir 3:266-271

Wulsten E, Lee G (2008) Surface temperature of acoustically levitated water microdroplets measured using infra-red thermography. Chem Eng Sci 63:5420-5424

Yarin AL, Brenn G, Kastner O, Tropea C (2002) Drying of acoustically levitated droplets of liquid-solid suspensions: Evaporation and crust formation. Phys Fluids 14:2289-2298

Zeng Y, Jiang L, Zheng W, Li D, Yao S, Qu JY (2011) Quantitative imaging of mixing dynamics in microfluidic droplets using twophoton fluorescence lifetime imaging. Opt Lett 36:2236-2238

Zhang J, Melton LA (1993) Potential systematic errors in droplet temperatures obtained by fluorescence methods. J Heat Transf 115:325-331

Zhao Y, Qiu H (2006) Measurements of multicomponent microdroplet evaporation by using rainbow refractometer and pda. Exp Fluids 40:60-69 